Revue d'histoire de l'Amérique française

REYUE D.HISTOIRE DE L'AMÉRIQUE FRANÇAISE

\title{
La bibliothèque du Collège des Jésuites
}

\section{Antonio Drolet}

Volume 14, numéro 4, mars 1961

URI : https://id.erudit.org/iderudit/302079ar

DOI : https://doi.org/10.7202/302079ar

Aller au sommaire du numéro

Éditeur(s)

Institut d'histoire de l'Amérique française

ISSN

0035-2357 (imprimé)

1492-1383 (numérique)

Découvrir la revue

Citer cet article

Drolet, A. (1961). La bibliothèque du Collège des Jésuites. Revue d'histoire de l'Amérique française, 14(4), 487-544. https://doi.org/10.7202/302079ar d'utilisation que vous pouvez consulter en ligne.

https://apropos.erudit.org/fr/usagers/politique-dutilisation/ 


\section{LA BIBLIOTHĖQUE DU COLLĖGE DES JÉSUITES}

\section{LES COMMENCEMENTS}

La Société de Jésus, fondée par Ignace de Loyola en 1534, avait établi des missions un peu partout dans le monde, moins d'un siècle plus tard. Les Jésuites furent les premiers missionnaires au Canada. C'est en 1611 qu'ils arrivèrent à Port-Royal, en Acadie, et ils s'établirent à Québec en 1625, à la demande des Récollets, arrivés à cet endroit depuis 1615. Ils y érigèrent une modeste résidence, sous le vocable de Notre-Dame des Anges, premier poste fixe de leur activité missionnaire à l'intérieur du pays. A la chute de Québec aux mains des Anglais, en 1629, ils quittèrent la ville, pour y revenir dès 1632 , lorsque le Canada fut rendu à la France. A partir de cette date, jusqu'en 1800, année de la mort du dernier Jésuite du régime français, les Jésuites eurent leur résidence principale à Québec, et c'est de ce centre qu'ils rayonnèrent à travers tout le continent nordaméricain par leurs randonnées missionnaires et leurs explorations et découvertes. En 1635, ils ouvrirent un Collège, premier établissement d'enseignement en Nouvelle-France, et le plus important sous le régime français. Il existe bon nombre d'ouvrages sur l'histoire des Jésuites au Canada et sur celle de leur Collège de Québec.

Il est normal de supposer que dès 1625 , les Jésuites eurent des livres en leur possession à Québec. C'est de 1632, cependant, qu'on peut établir sûrement les premiers commencements de leur bibliothèque, alors que les PP. Paul Lejeune et Charles Lallemant revinrent prendre leur poste. L'occupation de Québec par les Kirke avait été désastreuse pour leur premier établissement, qu'ils retrouvèrent en mauvais état. Voici ce qu'en écrit le P. Lejeune dans la Relation de 1632: ${ }^{1}$ 1632.

1 Brève relation du voyage de la Nouvelle-France fait au mois d'avril 
Les Anglais deslogeans, nous sommes rentrés dans notre petite maison. Nous y avons trouvé, pour tous meubles, des tables de bois telles quelles, les portes, fenestres, chassis, tous brisez et enlevés, tout s'en va en ruine, c'est encore pis en la maison des Récollets.

Les livres que les Jésuites avaient pu apporter avant 1632, ils les avaient sans doute pris avec eux pour retourner en France, et jusqu'à nos jours, il n'en a pas été retrouvé de cette première période. Comme pour le reste, ils ont dû recommencer à neuf leur bibliothèque, après leur retour. De fait, il existe encore quelques volumes dont on peut affirmer qu'ils furent dans leur établissement de Québec à partir de 1632. Ils sont marqués de l'inscription Mission des Jésuites en Nouvelle-France, datée de 1632. Il s'agit d'un volume de Sermons de saint Augustin, donné par Cramoisy, dont a parlé l'abbé Verreau ${ }^{2}$, de l'Anthologia Sacra, de Cressolles, et d'ouvrages de botanique de Dodoens, de Monardis et de Garcia de Orta. Ce sont là les ouvrages que l'on connaît des débuts de la bibliothèque des Jésuites à Québec.

Il ne s'agit que d'un petit nombre de volumes, qui faisaient partie d'un lot sans doute plus considérable, mais ils nous permettent déjà de nous faire une idée de la façon dont les Jésuites formèrent leur bibliothèque, dans quel esprit et à quelles fins.

Il n'est pas sans intérêt de noter la nature des œuvres de Monardis, Dodoens et Orta, botanistes, dont deux ont écrit sur les plantes de l'Amérique du Sud. Les Jésuites y eurent des missions dès le $16 \mathrm{e}$ siècle. La Nouvelle-France fut pour eux un nouveau champ d'apostolat, peu connu au début du $17 \mathrm{e}$ siècle. Pour se documenter sur le Canada, du moins autant qu'il était possible de le faire à une époque où les ouvrages sur le pays étaient encore très rares, les Jésuites apportèrent à Québec, des ouvrages qui pouvaient les renseigner sur la flore américaine. Ils voulurent avoir dans leur bibliothèque des auteurs qui pouvaient leur aider à connaître le pays et à tirer parti des ressources qu'il pouvait offrir. D'autres ouvrages manifestent qu'ils conservèrent ce souci de documentation.

2 Journal de l'Instruction Publique, (décembre 1868), 12:150-151. 
Les deux autres ouvrages conservés de la première époque, les Sermons de saint Augustin, une œuvre de Cressolles, ont leur place tout indiquée dans une bibliothèque religieuse. Ils portent une inscription prouvant qu'ils furent donnés par l'éditeur Cramoisy à la mission canadienne des Jésuites. Sébastien Cramoisy, directeur de l'Imprimerie Royale, éditeur de 41 des $4 a$ Relations des Jésuites, fut un bienfaiteur insigne de la bibliothèque du Collège des Jésuites.

Par ces quelques premiers ouvrages, il apparaît qu'elle fut formée avec une idée précise des services qu'on en attendait, et que de nombreux bienfaiteurs lui apportèrent leur contribution. Les donateurs furent particulièrement Cramoisy, des maisons des Jésuites en France, des auteurs et des personnages importants de la colonie.

Cramoisy apparaît au premier rang par le nombre d'ouvrages donnés. Il y a les deux ouvrages cités plus haut, puis les suivants: Philippaeus, Oseas (1632), Rossi, sur les Commentaires de S. Bonaventure (1646), Saussoy, Panoplia episcopalis (1646), Van den Steen, Commentaires sur l'Ecclésiaste (1639), Mascarenhas, Tractatus de sacramentis (1657), les Opera, de S. Jean Climaque (1633), Corneille de Lapierre, sur Josué (1642), la Théologie morale, de Gordon (1634), et plusieurs volumes des Relations. Un autre ouvrage donné par Cramoisy, une édition des Concordances de s. Augustin, éditée par sa maison en juin 1656, porte une note manuscrite, qui semble ouvrir un aperçu sur l'intérêt avec lequel on recevait au Collège de nouveaux ouvrages. Quelques lignes inscrites dans le volume précisent que l'ouvrage est arrivé à la bibliothèque le 7 juillet 1657, et semblent manifester la satisfaction de recevoir, si tôt bien et de si loin, une nouveauté de librairie. C'est une des rares notes, sinon la seule, qui révèle une appréciation personnelle, parmi des centaines d'inscriptions uniformes.

Suivant une coutume séculaire, les nouvelles entreprises missionnaires au 17ème siècle pouvaient compter sur l'assistance de maisons plus anciennes. Dans le cas de la Bibliothèque du Collège, on retrouve des preuves de cette aide dans des ouvrages donnés par différentes maisons des Jésuites de France. De 
celles de Paris, parvinrent les auteurs suivants: Jansenius (1593), Turrianus (1617), Velasquez (1640), Lorin (1605), Maractius (1633), Mariana (1620), Sanchez (1614), Suarez (1625), Alegambe (1643), Appianus (1692), Filliucci (1622), Laurent (1684). Du Collège de Metz, Gimignano (1550), de celui de Lyon, la Relation de 1659-60.

Des écrivains de la Société firent aussi envoi de leurs œuvres à Québec. Elles portent des dédicaces autographiées par leurs auteurs, lesquels les destinaient au Collège: Le Buffer (1632), Lejay (1725), Mariana, traduit par le jésuite Charenton (1725), Houdry (1712).

D'autres volumes sont des dons personnels de Jésuites, ainsi Hazeur, en 1699, donne un Beccanus; Glapion, Les Exercices de piété de Croiset.

Plusieurs personnages importants ont laissé une preuve de leur intérêt envers la bibliothèque, par des dons d'ouvrages. Le gouverneur Denonville fit don d'un Cassien (1682) ; l'intendant Duchesneau, un Nicole (1673), et un Corpus civilis de 1559, un Mornac (1646), Pline (éd. de 1585); le vicaire général Lauzon, un Mornay (1585) ; La Galissonnière, administrateur de la Nouvelle-France de 1747 à 1749, un Rozfeld (1583). Il existe aussi un bel in-folio, relié à ses armes, donné par l'intendant Talon, Saint Louys, du jésuite Lemoyne, accordé en prix à l'élève Noël Gaignon, comme l'atteste une inscription manuscrite du préfet des études, accompagnée du sceau du Collège.

Les dons ne suffirent évidemment pas à monter la bibliothèque. Sans doute faut-il y ajouter les livres que les missionnaires apportaient personnellement, en venant prendre leur poste au pays. On devait veiller en France à envoyer aussi les nouveaux ouvrages qui pouvaient avoir leur place dans une bibliothèque religieuse. Dans les plus anciennes Relations, les missionnaires sollicitent des dons de livres, surtout de dévotion, pour les malades de l'Hôtel-Dieu. ${ }^{3}$ Il n'apparaît pas cependant que c'est par ce moyen que les Jésuites demandaient et obtenaient des ouvrages pour leur propre maison. Il est possible qu'ils en

3 Thwaites, The Jesuit Relations, 49:211; 50:161; 51:115; 52:109. 
aient commandés aux libraires de Paris; les catalogues de libraires n'étaient pas inconnus en Nouvelle-France; il s'en trouvait parmi les livres de Guillaume Verrier, procureur à Québec de 1729 à $1759 .^{4}$

\section{BIBLIOTHĖQUE DE LA MISSION ET DU COLLĖGE}

La bibliothèque du Collège fut d'abord celle de la Mission des Jésuites au Canada. En fait, les inscriptions portent en grande partie le mot Collège, cependant il s'en trouve au delà d'une cinquantaine qui indiquent, Bibliothèque de la Mission des Jésuites de la Nouvelle-France, et dont la date d'édition est plus ancienne que 1672. Il s'agit évidemment des restes des tout premiers fonds de la bibliothèque. Quelques inscriptions désignent des ouvrages comme appartenant à des postes autres que Québec; l'une, de 1643, porte Mission de Saint-François-Xavier; ${ }^{5}$ un ouvrage de Montereul, La vie du Sauveur du Monde, conservé au Collège Sainte-Marie, de Montréal, a appartenu à la Mission de Tadoussac.

Il a été fait mention plus haut d'un ouvrage arrivé peu de temps après sa parution. Ce n'est pas le seul ouvrage qui soit entré à la Bibliothèque à une date rapprochée de sa publication. Dans un travail précédent sur les ouvrages de médecine à la bibliothèque du Collège de Québec, ${ }^{6}$ il a été relevé plusieurs de ces arrivages hâtifs. En voici quelques autres, qui ne touchent pas à la médecine: Sanroman, Expeditionum spiritualium libri v, paru en 1644, ex libris de 1646; Mascarenhas, Tractatus de sacramentis, 1656, ex libris 1657; Augustini, Concordantia, 1656, ex libris, 1657; Catrou, Histoire romaine, 1725, ex libris 1726; Bourdaloue, Conciones, 1714, ex libris, 1714 ; Parsons, Description de la vessie natatoire, 1743, ex libris, 1745 . Il en existe d'autres dont l'intervalle entre la date d'édition et celle de l'ex

${ }^{4}$ Inventaire des biens de Me Guillaume Verrier. Ms. Archives du Séminaire de Québec.

5 Il y eut apparemment deux postes missionnaires de ce nom: l'un, près de Montréal, l'autre à la Baie des Puants (Green Bay), sur la côte ouest du Lac Michigan. Cf., Rochemonteix, Les Jésuites en Nouvelle-France au $18^{e}$ siècle (Paris, 1906), 483, 484, et Thwaites, op. cit., 54:197; 56: 19.

6 Laval Médical, (mai 1957), 22: 688-699. 
libris est un peu plus long, deux à quatre ans; on peut dire qu'ils étaient encore des nouveautés d'édition à leur arrivée à Québec, si on se rappelle que les envois de France à Québec ne se produisaient d'ordinaire qu'une fois l'an.

\section{CATALOGUES DE LA BIBLIOTHËQUE}

Le pillage des maisons des Jésuites en France en 1763, l'occupation militaire du Collège de Québec, à partir de 1759 , et la dilapidation qui s'ensuivit, expliquent que les documents sur la Bibliothèque du Collège sont pratiquement inexistants ou inconnus.

Dans le Journal des Jésuites, on parle, à l'occasion, de livres prêtés et donnés en cadeaux. Les livres de la bibliothèque circulaient certainement entre les mains des gens de l'extérieur. ${ }^{7}$ La Bibliothèque fut la première importante à Québec, et on devait y recourir à l'occasion pour la valeur et le nombre des ouvrages qui s'y trouvaient. En date du 6 mars 1645, le Journal rapporte que l'« on prête deux livres à Mons. Nicolet, qui s'en va à l'Isle aux Oyes, la vie de J.C. et l'abrégé de Dupons ». Pour le premier de l'an, suivant une coutume française, les Pères font des cadeaux sous forme de livres, à diverses personnes religieuses et laïques. Le Journal en indique pour les ler janvier de $1645,1647,1648,1649,1650$ et 1651 . Ces dons se faisaient sans doute sans entamer les ouvrages de la bibliothèque, et prouvent qu'on en possédait suffisamment d'autre part pour se permettre certaines générosités.

Bien qu'on n'en ait rien retrouvé, on peut affirmer qu'il a certainement existé un catalogue ou registre des livres de la bibliothèque. C'était un usage du temps, comme en fait foi le Catalogue de la Bibliothèque du Séminaire de Québec, dressé au 18ème siècle et conservé aux archives du Séminaire.

En ce qui regarde les livres des Jésuites, l'inscription Inscrit au Catalogue, répétée soigneusement une fois et souvent deux, sur la page-frontispice d'un si grand nombre de volumes, peut

${ }^{7}$ Antoine Roy, Les Lettres, les Sciences et les Arts au Canada sous le Régime français (Paris, 1930), 63. 
être considérée comme une preuve suffisante de l'existence d'un catalogue. Une première inscription datée était faite à l'entrée du livre à la bibliothèque. De plus, la plupart des ouvrages portent deux inscriptions datées de 1720 à 1745, en exceptant, pour la première date, les ouvrages parus après 1720 . La répétition régulière de ces deux inscriptions indiquerait qu'on procéda en 1720 et en 1745 à refaire la liste du contenu de la bibliothèque. On connaît au moins un bibliothécaire, par son nom, le religieux Laure, novice, en fonction en $1716 .^{8}$

\section{LA BIBLIOTHĖQUE APRÈS 1759}

La prise de Québec en 1759 , le traité de 1763 , marquèrent définitivement le sort du Collège de Québec, en même temps que celui de l'Ordre au Canada. Le maintien de l'Ordre fut refusé et interdit, par l'acte de reddition de la ville de Québec et par le traité passé quatre ans plus tard. Le Collège subit les premiers coups dès 1759 , alors qu'il fut réquisitionné par l'armée anglaise. Les archives et la bibliothèque furent pillées et dispersées, une première fois en 1763 , et plus complètement encore en $1773 .^{9} \mathrm{En}$ 1776, le Collège fut complètement fermé. Les salles de classes furent converties en salles d'audience, en dépôts d'archives, en magasin de vivres et en prison. On ne laissa que la chapelle et quelques chambres à l'usage des derniers religieux. ${ }^{10}$

Le pillage et la dispersion subis par le Collège et ce qui s'y trouvait expliquent que peu d'années plus tard, certains ouvrages qui en provenaient furent mis en vente par le Quebec Printing Office, atelier d'imprimerie et librairie, comme en fait foi l'étiquette de cette maison, portant des prix de vente, qu'on trouve sur certains volumes. D'autres portent des marques de propriété de personnes vivant avant 1800, preuve qu'elles les eurent en leur possession dès cette époque. Il est évidemment impossible d'apprécier la proportion de la bibliothèque qui échappa à la dispersion.

\footnotetext{
8 Thwaites, op. cit., 68: 326, en note.

9 Thwaites, op. cit., 71: 120. II $: 208$.

10 Rochemonteix, Le Collège des Jésuites de Québec au $18^{e}$ siècle,
} 


\section{LES BIENS DES JÊSUITES}

Dès le traité de 1763 , la question des biens des Jésuites a commencé de se poser, pour ne trouver une solution définitive que plus d'un siècle plus tard. A partir de la Conquête, l'intention du gouvernement anglais, travaillé par diverses influences, n'apparut que trop évidente. Les Jésuites canadiens, déjà réduits à un petit nombre, furent tour à tour frappés par les inconvénients de l'âge avancé. Ils s'efforcèrent cependant de distribuer les biens dont ils avaient la garde, en tenant compte des fins pour lesquelles ces biens avaient été donnés et conservés. Une lettre du Père Glapion, du 31 décembre 1789, est révélatrice à ce sujet. ${ }^{11}$ Elle contient une renonciation des quatre Jésuites survivants en faveur des «citoyens canadiens 》 à 《toute propriété et concession » leur appartenant comme membres de leur Ordre. Cette renonciation fut faite à condition que le dernier d'entre eux, jouirait jusqu'à sa mort, de différents biens, entre autres et nommément « de leur bibliothèque ». La valeur et le sens de cette renonciation, qui n'eut pas de suite, ont été discutés ; elle établit du moins, qu'à cette date, la bibliothèque existait encore comme un tout, plus ou moins entamé, mais conservée dans une des salles du Collège.

\section{LE PÈRE CASOT}

N'ayant pu réussir à s'assurer qu'après leur mort leurs biens ne seraient pas saisis par le Gouvernement, les Jésuites s'efforcèrent d'en distraire le plus possible, du moins pour les biens meubles, en les distribuant au moment opportun aux personnes et aux institutions de leur choix. Après la mort du P. Weil, dernier jésuite résidant à Montréal, le P. Casot s'y rendit pour distribuer tous les biens meubles de leur Maison à cet endroit, ${ }^{12}$ et les faire ainsi échapper à la mainmise du Gouvernement. Luimême, à Québec, il distribua objets du culte, meubles, livres, manuscrits à différentes institutions comme l'Hôtel-Dieu, le Séminaire de Québec, l'Hôpital Général, et à des particuliers, ainsi qu'il apparaît par des livres qui portent leurs noms, et qui

11 Thwaites, op. cit., 71: 100 ss.

12 Thwaites, op. cit., $28: 305 ; 71: 395$. 
provenaient de la bibliothèque du Collège. Cette précaution permit à l'Hôtel-Dieu de conserver des manuscrits importants qui furent plus tard, en 1843, remis au P. Félix Martin, supérieur et fondateur du Collège Sainte-Marie, à Montréal. ${ }^{13}$

En ce qui regarde la bibliothèque, des documents et des inscriptions dans des volumes, manifestent le soin que se donna le P. Casot pour faire bénéficier les maisons religieuses des biens dont il disposa avant sa mort, survenue le 16 mars 1800, à l'HôtelDieu de Québec.

Les livres de comptes du Séminaire de Québec portent une entrée en juin 1797, qui se lit ainsi: Payé à deux charretiers pour le transport de la Bibliothèque des Jésuites au Séminaire, $3 \mathrm{sh}^{{ }^{14}}$ Un passage d'une lettre de Mgr Joseph-Octave Plessis confirme ce fait en rapportant que le $\mathrm{P}$. Casot donna en 1797 la bibliothèque au Séminaire. ${ }^{15}$

Cependant, ce dont le P. Casot avait ainsi disposé en faveur du Séminaire, ce n'était pas tout ce qui restait de la Bibliothèque. Déjà, comme nous l'avons indiqué, des parties plus ou moins importantes avaient disparu du Collège, pour se retrouver en d'autres mains, comme en font foi divers ex libris; ainsi, un Aretha (1532), un Saint Justin (1551), sont marqués de la griffe de J.-O. Plessis, datée de 1750 ; les Sermons de Texier (1675) portent la marque de possession de G. Genest, en date de 1794 ; J. L. Borgia indique 1799 comme la date de son ex libris d'un Cornelius Nepos (1569), et des Satires de Juvénal (1658); Ranvoyzé, en 1797, possède le Triomphe de saint Augustin de Du Bosc; Mgr Hubert, mort en 1797, possédait un Terentius (1641). Tous ces ouvrages portaient déjà l'ex libris original et plus ancien de la bibliothèque du Collège. Ils en ont été distraits, par don ou autrement, avant que le P. Casot ne dispose de l'ensemble. Combien d'autres ont pu connaître des avatars semblables?

13 Thwaites, op. cit., $28: 305$.

14 Livres de compte, 1797. Archives du Séminaire de Québec.

15 Ivanhoë Caron, «Inventaire de la correspondance de Mgr JosephOctave Plessis, évêque de Québec, 1797-1825 », Rapport de l'Archiviste de la province de Québec, (1927-28). Lettre de Mgr J.-O. Plessis à Mgr Denault, 24 mars 1800, 222. 
De plus, ce n'est pas seulement au Séminaire que le P. Casot distribua des livres. Dora Hood, bouquiniste et bibliophile de Toronto, mentionne un volume des Relations des Jésuites, portant la marque du Collège et l'autographe de John Neilson, ${ }^{16}$ ami du dernier Jésuite du régime français. Le P. Casot lui aurait aussi fait don d'une centaine de volumes qui se trouveraient maintenant au Scolasticat de l'Immaculée-Conception à Montréal.17 Une tradition veut que certains biens et meubles du P. Casot aient été conservés par les descendants de John Neilson. A la mort du dernier du nom, les documents et livres qu'il avait hérités de son ancêtre furent dipersés dans des ventes. Une partie en fut acquise par les Archives canadiennes et celles de la province de Québec. S'y trouvait-il d'autres livres des Jésuites ?

Une maison religieuse ancienne, l'Hôpital Général de Québec, reçut un bon nombre d'ouvrages du P. Casot lui-même. Suivant les inscriptions qu'ils portent, c'est pour l'infirmerie, quant aux ouvrages de médecine, et pour l'aumônier de l'Hôpital, que le P. Casot destina ces dons. Ils sont encore conservés et l'archiviste de l'Hôpital, Sœur Saint-Alphonse, nous a permis d'en prendre connaissance et d'en dresser la description. ${ }^{18}$

Les différents dons du P. Casot, faits avant sa mort, n'avaient cependant pas épuisé complètement ses possessions en livres. Il en avait conservé pour son usage, et ils se trouvaient parmi les biens qu'il laissa et qui furent saisis après son décès, au nom du Gouvernement, le 16 avril $1800 .{ }^{19}$ Il en est décrit environ deux cents dans l'inventaire du mobilier dressé par le Shérif de Québec. Les titres abrégés en sont donnés, ce qui a permis de les faire entrer nommément dans notre reconstitution.

Comme l'écrivait jadis l'abbé Verreau, des livres des Jésuites, il s'en trouve dispersés un peu partout. A l'Hôtel-Dieu de

16 Dora Hood, The Side Door (Toronto, 1958), 99.

17 Marius Barbeau, Trésor des Anciens Jésuites (Ottawa, 1957), 29.

18 On trouve dans le Bulletin des Recherches historiques, (janvier 1938), 17 et ss., un article du P. Léo Hudon, s.j. où sont relevés un certain nombre de ces ouvrages.

19 J.-E. Roy, «Un document historique », Revue Canadienne, (1891), $3^{\mathrm{e}}$ s.: 25:271-282. Rapport du Comité de la Chambre d'Assemblée (1824), 232. 
Québec, on en connaît quelques-uns, ainsi qu'à la Résidence des Jésuites de la rue Dauphine, à Québec, et à leur Collège SaintCharles Garnier. La Maison Mère des Sœurs de la Charité en possède trois volumes. Dès leur retour au Canada, en 1842, les Jésuites n'ont pas négligé d'en recueillir, qui sont conservés au Collège Sainte-Marie, à Montréal, ainsi qu'à la maison de l'Immaculée-Conception. La Mission iroquoise de Caughnawaga en conserve depuis longtemps. Les collectionneurs s'intéressent depuis toujours, pourrait-on dire, aux livres des Jésuites. Nous avons vu qu'ils ont commencé à le faire dès la fin du dix-huitième siècle. Il s'en trouverait même dans la célèbre bibliothèque de Pierpont Morgan, aux États-Unis. ${ }^{20}$

On peut déduire facilement de tout ce dispersement que la reconstitution intégrale de la Bibliothèque des Jésuites est une entreprise chimérique. Dans un effort plus modeste, qui réalise le désir exprimé autrefois par l'abbé Verreau, ${ }^{21}$ et par d'autres depuis, j'ai tenté une reconstitution qui permet de connaître au moins une partie d'un tout dont l'importance échappe aux chercheurs. Cette partie atteint près de 700 ouvrages, comprenant près de 1000 volumes.

\section{TRAVAUX SUR LA BIBLIOTHĖQUE}

L'abbé Verreau, suivant toute apparence, fut le premier à parler de la bibliothèque des Jésuites, dans un article du Journal de l'Instruction publique, déjà cité. Joseph-Edmond Roy a reproduit le document qui contient l'inventaire des biens laissés par le P. Casot. Dans une étude, Les bibliothèques canadiennes (1916), Aegidius Fauteux a répété à peu de choses près, ce qu'en avait écrit l'abbé Verreau. M. Antoine Roy ${ }^{22}$ cite Fauteux et ajoute, à propos de la bibliothèque du Séminaire, «pour ancienne qu'elle fut, cette bibliothèque avait pu s'enrichir des dépouilles des Jésuites ». Comme nous l'avons vu, ce n'est qu'en 1797, que le Séminaire reçut les livres des Jésuites. Le P. Léo Hudon, dans

20 Dora Hood, op. cit., 33.

21 Journal de l'Instruction Publique, loc. cit.

22 Antoine Roy, op. cit., 67. 
un article du Bulletin des Recherches historiques ${ }^{23}$ parle des ouvrages qui se trouvent à l'Hôpital Général de Québec. Dans le Canadian Council Bulletin a paru un article sur la bibliothèque, où, pour la première fois, on faisait connaître la composition des fonds connus de cette bibliothèque. ${ }^{24}$

Deux articles ont paru, l'un dans le Naturaliste canadien, ${ }^{25}$ l'autre, dans le Laval Médical, ${ }^{26}$ sur les ouvrages scientifiques et médicaux de la bibliothèque. M. Marius Barbeau ${ }^{27}$ a fait connaître différents endroits et bibliothèques où se trouvent des livres des Jésuites.

Tous ces travaux ont contribué à faire mieux connaître la bibliothèque, sans donner une idée exacte de ses fonds, faute de connaissance précise des ouvrages qui en subsistent. Il serait peut-être possible de s'en faire une idée approximative par la connaissance d'une bibliothèque de l'époque, dans une maison religieuse du même ordre en Europe. Encore que les catalogues de ce genre de bibliothèques ne soient pas courants, il convient d'observer que la Bibliothèque du Collège de Québec, établie en un poste de mission dans un pays neuf, n'a peut-être pas eu le même caractère qu'une bibliothèque ordinaire de collège en France. Il ne s'agissait pas seulement d'un collège, mais aussi de quelque chose de différent, c'est-à-dire, un poste central de mission dans un continent éloigné par des semaines de voyage de la civilisation. Ce poste, ce collège devaient se suffire à euxmêmes, en plus de servir de centre à des postes encore plus éloignés dans la sauvagerie. Rochemonteix a bien décrit la fonction de cette maison de Québec:

Quant au collège, il était, pour les jeunes religieux de la compagnie nouvellement arrivés de France, une école et une préparation aux missions sauvages; ils y apprenaient la langue du pays, ils s'instrui-

${ }^{23}$ Léo Hudon, «Souvenirs du vieux Collège de Québec 》, Bulletin des Recherches historiques (janvier 1938), 17-25.

24 Canadian Library Council Bulletin, (juillet 1945), I: 49-50.

25 « Ouvrages scientifiques de la Bibliothèque du Collège des Jésuites 》, Le Naturaliste canadien, (avril-mai 1949), 82:102-107.

26 « Les ouvrages de médecine à la résidence des Jésuites de Québec 》, Laval Médical, (mai 1957), 22: 688-699.

27 Marius Barbeau, op. cit., 229-231. 
saient, auprès des vieux apôtres de la NouvelleFrance, des mœurs et des habitudes des Indiens, de toutes les industrieuses inventions de la charité pour les convertir et les attacher à Dieu; ils faisaient ou terminaient leurs études théologiques, et, pendant ce temps, ils professaient ou ils surveillaient. ${ }^{28}$

La formation de la bibliothèque se ressentit nécessairement de la fonction importante de la résidence de Québec. On tâcha de se procurer les ouvrages nécessaires aux besoins multiples de la vie dans un milieu nouveau et où tout était à faire.

Le collège fut une résidence de missionnaires, un collège classique, un séminaire de théologie, il logea l'apothicairerie la plus importante de la Nouvelle-France; on y pratiqua des recherches scientifiques, particulièrement sur la flore, et il fut même question d'y établir un observatoire astronomique. Comme résidence missionnaire, on devait y trouver ce qui pouvait renseigner sur les entreprises d'évangélisation, tant au point de vue spirituel que matériel. Les missionnaires tâchaient de subvenir eux-mêmes aux travaux nécessaires. C'est un Frère jésuite, qui dirigea la construction du Collège de Québec, un édifice remarquable pour l'époque. Le premier établissement enropéen permanent en Ontario, c'est à la Mission Sainte-Marie qu'il fut érigé par les Jésuites. Les découvertes archéologiques ont montré l'importance des constructions de ce poste. ${ }^{29}$

L'enseignement du collège comprenait les différentes connaissances qui faisaient partie des études classiques à l'époque, en plus de la théologie. On ajouta à ces connaissances, d'autres disciplines exigées par les besoins d'un pays à explorer et à mettre en valeur: l'arpentage, la navigation, l'architecture, la construction des navires, la métallurgie. On peut rappeler, à ce sujet, le cas de Joliet, il étudia au collège la musique, l'arpentage et l'astronomie, ces deux dernières connaissances lui furent nécessaires dans ses explorations, et il les enseigna lui-même par la suite.

\footnotetext{
28 Rochemonteix, Les Jésuites et la Nouvelle-France au XVIIe siècle, (5 vol., Paris, 1895), I: 221.

29 Kenreth E. Kidd, The excavations of Sainte-Marie. I, (Toronto, University of Toronto Press, 1949).
} 
Une science fut particulièrement en honneur chez les Jésuites de la Nouvelle-France, la médecine. Sa nécessité ne pouvait échapper à des gens qui s'isolaient loin de tous secours autres que ceux qu'ils pouvaient connaître eux-mêmes. Le renom de plusieurs d'entre eux est parvenu jusqu'à nos jours pour leur habileté dans l'art médical: Simon Baron, chirurgien, le Frère René Goupil, chirurgien, compagnon du P. Jogues, martyrisé et canonisé avec lui, Noël Juchereau, infirmier et apothicaire, le Frère Bonnemer, infirmier, le Frère Jean Boispineau, apothicaire et chirurgien, le $\mathrm{P}$. Farault, attaché à la Mission de Lorette. ${ }^{30}$ $\mathrm{Au}$ Collège se trouvait le dépôt général des remèdes pour la colonie. ${ }^{31}$ Cette activité médicale fait comprendre l'utilité du grand nombre d'ouvrages de médecine de la bibliothèque, près de 150 , dont plusieurs portent l'inscription précise: Pharmacopée. C'est le groupe d'ouvrages sur la médecine le plus important sous le régime français.

D'autres sciences étaient aussi en honneur au Collège. Le P. Joseph-François Lafitau, missionnaire au Sault-Saint-Louis, près de Montréal, découvre le ginseng au Canada, sur lequel il rédige un mémoire. Un "Traité des animaux, des oiseaux, des poissons » aurait eu pour auteur un jésuite. ${ }^{32}$

Lorsque Michel Sarrazin fait des recherches sur les propriétés de plantes canadiennes, il est assisté d'un frère apothicaire. ${ }^{33}$ On enseignait l'hydrographie au Collège, science qui comprenait aussi l'astronomie. Le P. de Bonnécamps, envoyé à Québec expressément pour cet enseignement, projeta l'érection d'un observatoire astronomique. Il fit des observations qui parurent dans les Mémoires de Trévoux, en mars $1747 .^{34}$

${ }^{30}$ Charlevoix, Histoire de la Nouvelle-France, (6 vol., Paris, 1744), I: 337. - M. J. \& Geo. Ahern, Notes pour servir à l'histoire de la médecine dans le Bas-Canada (Québec, 1923), 57.

31 Maude E. Abbott, History of medicine in the province of Quebec (Toronto, MacMillan, 1931), 28.

32 Arthur Vallé, Un biologiste canadien. Michel Sarrazin, 1659-1735 (Québec, 1927), 85 ss.

33 Id., 58.

34 A. E. Gosselin, L'Instruction au Canada sous le régime français (Québec, 1911), 251. - A. Gosselin, «Les Jésuites au Canada. Le P. de Bonnécamps 》, Mémoires de la Société Royale du Canada, $2^{\mathrm{e}}$ s., (1895), I, sect. 1: 27-28. - Antoine Roy, op. cit., 139. 
Ces quelques notes n'ont pour but que de rappeler les entreprises diverses auxquelles s'adonnèrent les Jésuites au Canada, missionnaires, hommes de science, éducateurs; ces intérêts ont certainement eu leur influence sur le choix des ouvrages qu'ils voulurent avoir dans leur bibliothèque.

\section{COMPOSITION DE LA BIBLIOTHÈQUE}

De fait, la composition de la bibliothèque, les ouvrages qui s'y trouvaient, nous présentent, sous un autre aspect, la variété des préoccupations de ses possesseurs, au moins par ce qui nous en est parvenu. Et doit-on supposer que certaines parties ont plus que d'autres souffert des événements. Il est remarquable, par exemple, que des ouvrages historiques sur le Canada, parus au $17 \mathrm{e}$ et $18 \mathrm{e}$ siècles, qui devaient nécessairement se trouver dans la bibliothèque, n'ont pas été retrouvés. Sans doute, ont-ils très tôt attiré l'attention des collectionneurs pour leur intérêt particulier.

Quoi qu'il en soit des ouvrages disparus, dispersés, devenus introuvables, en se limitant à ce qui est connu par notre reconstitution, voici un tableau qui en résume le contenu d'après les matières traitées:

Ouvrages religieux

Médecine

Belles Lettres

Droit

Histoire et géographie

Journaux

Philosophie

Sciences

(Mathématiques, Physique, Chimie, Botanique, Astronomie, Navigation)

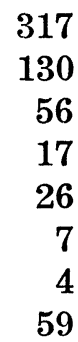

6

Ce tableau indique la spécialité des ouvrages, et non le nombre de volumes.

La bibliothèque contenait des œuvres parues aux 15e, 16e, $17 \mathrm{e}$ et $18 \mathrm{e}$ siècles. Le 15 ième siècle est représenté par un seul 
ouvrage, une édition des Legenda Sanctorum, de Voragine, éditée en 1483. Pour le 16ème siècle, ils sont au nombre de 35, dont l'un est de grande valeur, par sa provenance de la bibliothèque de Jean Grolier, premier bibliophile français. Il s'agit de Ortus deliciarum, sous la reliure originale de Grolier, marquée de sa célèbre devise: Portio mea, Domine, sit in terra viventium. Pour le reste, la bibliothèque est des $17 \mathrm{e}$ et $18 \mathrm{e}$ siècles. C'est d'ailleurs au cours de ce dernier siècle que la bibliothèque s'est augmentée le plus. En plus d'un catalogue, la bibliothèque devait avoir un certain classement. On trouve, sur un grand nombre de livres, des lettres de l'alphabet qui devaient marquer leur place respective dans l'ensemble.

\section{CONCLUSION}

Le catalogue qui suit ces notes, représente ce qu'il nous a été possible de retracer de la Bibliothèque du Collège des Jésuites de Québec. Elle n'y est certainement pas au complet; il a existé sans doute d'autres ouvrages, ils sont soit disparus, ou encore enfouis dans des collections où leur caractère particulier n'a attiré qu'une attention passagère, sans qu'on ait entrepris de les noter spécialement.

Notre travail permet au moins de se faire une meilleure idée de la première bibliothèque canadienne. Elle fut une importante collection d'ouvrages à l'époque où la culture européenne fut apportée par les pionniers du pays. On a dit que le Canada fut, en fait, une province de France sur le continent américain. La civilisation qu'on y établit venait d'un pays qui tenait alors la tête du monde civilisé. Les ouvrages de la bibliothèque des Jésuites peuvent aider à faire connaître l'ambiance, les conditions intellectuelles de cette civilisation qui était celle des Français du Canada aux 17ième et 18ième siècles.

Québec.

ANTONIO DROLET 


\section{NOTES EXPLICATIVES SUR LE CATALOGUE}

La description bibliographique des ouvrages est suivie de divers renseignements.

a) Les dates inscrites à droite, à la suite de la description bibliographique, sont celles des ex libris que l'on trouve dans les ouvrages. Un bon nombre des livres ont reçu deux inscriptions différentes, avec dates différentes; lorsqu'une inscription n'est pas datée, elle est indiquée par l'abréviation: s.d., sans date.

b) L'inscription la plus fréquente, portant Collège de Québec, n'a pas été reproduite; lorsque l'inscription est différente, elle a été répétée, p.e.: Miss. Nov. Franc.

c) Un bon nombre des ouvrages se trouvent à la Bibliothèque de l'Université Laval; dans leur cas, la description est simplement suivie d'une date ou de deux, suivant le cas.

d) Pour les ouvrages qui n'appartiennent pas à la Bibliothèque de l'Université Laval, on a indiqué leur appartenance, soit par le nom de leur propriétaire, soit par un symbole.

e) La Lettre C sert pour indiquer les ouvrages décrits dans l'inventaire des biens du P. Casot.

f) La Lettre $B$ se rapporte aux ouvrages mentionnés dans l'ouvrage de Marius Barbeau, Trésor des Jésuites.

g) Les ouvrages qui se trouvent aux archives de l'Hôpital général de Québec, sont indiqués par Hôp. Gén.

h) Ceux qui appartiennent aux Archives du Collège SainteMarie de Montréal, sont suivis de la mention: Collège Sainte-Marie.

i) Miss. Nov. Franc. signifie Mission de la Nouvelle-France.

\section{LISTE DES ABRÉVIATIONS}

S.d.: sans date, dans le cas d'un ex libris non daté.

Hôp. Gén.: Hôpital Général de Québec.

Miss. Keb. Nov. Franc.: Mission de Québec en NouvelleFrance.

Pharmac.: Pharmacopée.

Res. Marian.: Résidence des Jésuites à Montréal.

Miss. Huro.: Mission des Hurons.

Miss. Tad.: Mission de Tadoussac.

Miss. Nov. Franc.: Mission de la Nouvelle-France. 


\section{CATAlOGUE DE LA BIBLIOTHĒQUE DU COLLEGE DES JÉSUITES DE QUÉBEC \\ 1632-1799}

\section{ESSAI DE RECONSTITUTION}

1 Abbé de la Trappe

Réponse au traité des études monastiques. Paris, 1692,

2 Abelly, Louis, 1603-1691.

Medulla theologica ... Paris, 1654.

3 Abelly, Louis.

(Dupl.) s.d./1745

La vie du vénérable serviteur de Dieu Vincent de Paul, instituteur et premier supérieur général de la Congrégation de la Mission. Paris, 1664.

s.d. $/ 1745$

Hôp. Gén.

4 Abrégé de géographie.

C

5 Académie royale de chirurgie. Mémoires... Paris, 1743. 3 vol.

6 Acquiaviva, Claude, 1543-1615.

Epistolæ duæ ... De officii divini recitatione, ac celebratione missæ. Monita complectens, formansi concionatoribus accommodata. Vurdigalæ, 1614.

s.d.

7 Actions chrétiennes.

C

8 Agneau pascal ou explication des cérémonies... en la manducation de l'agneau de Pasque... Cologne, 1686.

9 Alegambe, Philippe, s.j., 1592-1651.

$1720 / 1745$

Bibliotheca scriptorum Societatis Jesu post... catalogum R.P. Petri Ribadeneiræ, s.j.... Anvers, 1643. (Domus probdis paris. Soc. Jesu)

10 Alvares de Paz, I., s.j., 1560-1620.

Opera. Moguntiæ, 1614-1629. 3 vol.

$$
\text { s.d./1745 }
$$

11 Amaltheus prosidicum, sive brevis et accurata ... prosodia ... Ed. 3a. Lyon, 1663.

12 Id. (ex. double)

13 Amand, Pierre, 1650-1720.

Nouvelles observations sur la pratique des accouchements . . . 2e éd. Paris, 1715.

$1720 / 1745$ 
14 Analyse démontrée ou la méthode de résoudre les problèmes des mathématiques et d'apprendre facilement les sciences. Paris, 1708. 2 vol.

15 Andry de Boisregard, Nicolas.

1745

Remarques de médecine sur différents sujets... Thèse... que l'homme vient d'un vers ... 2de éd. Paris, 1711.

16 Année du chrétien. 13 vol.

$\mathrm{C}$

17 Apologie pour la doctrine des Jésuites envoyée à $\mathrm{Mgr}$ d'Arras à l'occasion de la censure qu'il a faite du livre d'un calviniste allemand. Liège, 1703.

18 Id. (Ex. double).

1720

19 Apologie pour les casuistes contre les calomnies des jansénistes... Paris, 1657.

20 Appianus, Alex.

Rom. historiarum ... Paris, 1592.

$1720 / 1745$

21 Arbuthnot, John, 1667-1735.

Essai sur la nature et le choix des aliments... Trad. de l'anglois. Paris, 1741.

22 Aretha, Cæsareæ.

1745

... Collectio expositionum ex diversis sanctis viris in Joannes... Apocalypsim. Verone, 1532.

23 Argentré, Bertrand d', 1519-1590.

$1720 / 1745$

Plessis, 1790.

Commentarii in patrias Britonum leges... Ed. 8a. Anvers, 1664. 1745

24 Argentré, Bertrand d' Coustumes générales... de Bretagne. Reformées en l'an 1580. Paris, 1613.

25 Argole, André, 1570-1650.

Ephemeri des juxta Tychonis hypotheses et e cœlo deductas observationes. Ab anno 1671 ad 1700. Lyon, 1659.

26 Aristote

Ethicorum ad Nicomachum libri decem. Paris, 1542.

27 Id.

Sententiæ. Paris, 1574.

28 Arnauld, Antoine, 1560-1619.

1745

L'esprit de Mr Arnaud tiré de sa conduite et des écrits de luy et de ses disciples. Deventer, 1684. (Inter prohibitos libros).

3.d./1745

29 L'art de bien vivre et de bien mourir... Vannes, 1704. 
30 L'art de naviguer démontré par principes et confirmé par plusieurs observations tirées de l'expérience. Paris, 1677.

31 De l'art de saigner, accommodé aux principes de la circulation sanguine... 2de éd. Paris, 1689.

32 Athanase, $\mathbf{S}$.

$1720 / 1745$

(Athanasiou dialogi V.) 1568.

33 Id.

Opera omnia. $1601.2 \mathrm{v}$.

34 Aubignac, François Hedelin d', 1604-1676.

La pratique du théâtre. Amsterdam, 1715.

1745

35 Augustin, S.

Concordantiæ augustinianæ... labore F. Davidis Lenfant. Paris, 1656.

Colleg. Kebec. 7 ju. 1657 1745

36 Id.

Confessions.

C

37 Id.

Milleloquium veritatis. Paris, 1649. 2 vol.

38 Id.

Sermons.

s.d./1745

d.

Miss. Keb. Nova. Franc. 1632

38a Id.

Sermons. 1561.

(Ex dono Pni de Montmagny moderatoris nostri.)

39 Aux Cousteaux, Arthur.

Missa quinque vocum ... Paris, 1647.

40 Id.

s.d.

Octo cantica Divæ Mariæ Virginis ... Paris, 1641.

41 Avancin, Nicolas

Vita et doctrina Jesus-Christi ex quatuor evangelistis collecta ... Paris, 1695.

1745

Hôp. Gén.

42 Avis donné aux confesseurs.

C

43 Arucci, Vincent

Rituario. Roma, 1611.

44 Bagot, Jean, s.j., 1580-1664.

s.d./1745

Apologeticus fidei ... Paris, 1644-45. 2 vol.

45 Bail, Louis, -1669.

s.d./1745

La théologie affective ou saint Thomas en méditation. Paris, 1659. 
46 Baronius, César, card., 1538-1607.

Martyrologium romanum ... Paris, 1613.

47 Barradas, Sébastien, s.j., 1542-1615.

s.d./1745

Commentarii in concordiam et historiam evangelicam... Lyon, 1621. 2 vol.

48 Barry, Paul de, s.j., 1585-1661.

Miss Nov. Franc. s.d./1745

Les méditations de Philagie pour tous les jours de l'année... Lyon, 1649.

49 Id. 1745

La solitude de Philagie... exercices spirituels... 4e éd. Lyon, 1645.

50 Bauderon, Brice, 1540-1625.

1745

Pharmacopée... Lyon, 1655.

Pharmacop. Coll. Keb., s.d.

51 Bauldry, Michel 1720

Manuale sacrarum cæremoniarum ... Paris, 1646.

52 Bauny, Etienne, s.j., 1564-1649.

s.d./1745

Pratique du droit canonique ... Paris, 1633.

53 Id.

Theologia moralis. Paris, 1645-47. 2 vol.

54 Becan, Martin, s.j., 1550-1624.

Summa theologiæ scholasticæ. Paris, 1634.

55 Becan, Martin

Summa theologiæ scholasticæ... Paris, 1666. (Dedit Collegio Kebec, P. Hazeur, 1699.)

- Ex. double

$1699 / 1745$

56 Bellegambe, François

Enchiridion theologopracticum ... de jubilaeo ecclesiastico. Lille, 1699.

57 Bellecoste, $\mathrm{M}$.

s.d./1745

Le chirurgien d'hôpital. 3e éd. Paris, 1734.

1745

Apothicairerie Hôp. Gén.

58 Belsunce de Castelmoron, Henri-François-Xavier de, s.j., 1671-1755. Instruction pastorale sur l'incrédulité. Marseille, 1754

59 Benzon, Rutilius

Dissertationes et commentaria in beatissimæ Virginis canticum Magnificat, salutationem angelicam ... Douay, 1626

60 Berenger, W.

Celandre ou traité nouveau des descentes ... Paris, 1695. 
61 Bernard, S.

Opera omnia. Anvers, 1620.

62 Id.

s.d./1745

s.d./1745

Ranvoyzé, 1800

63 Bernier, Jean, 1625-1688.

Histoire chronologique de la médecine . . . 2e éd. Paris, 1717.

64 Bernières-Louvigny, Jean de $1720 / 1745$

Le chrestien intérieur... Paris, 1663.

Res. Marian.

$1721 / 1745$

65 Id.

Hôp. Gén.

Les œuvres spirituelles . . . Paris, 1670.

s.d./1745

66 Béruelle, Pierre de, card., 1575-1629.

Hôp. Gén.

Oeuvres ... Paris, 1657.

Miss. Nov. Fr. 1745

67 Besson, Jean.

In canticum canticorum Salomonis novæ lucubrationes... Toulouse, 1646 .

68 Bezanson, Germain de

Miss. Nov. Fr. 1745

La médecine prétendue réformée ou l'examen d'un traité des fièvres ... Paris, 1683.

69 Id.

Nouveau traité des fièvres. Paris, 1691.

70 Bible

C.

71 Bible. N.T. Grec.

Novum testamentum. Anvers, 1584

72 Bible. Hébraïque.

s.d./1745

Biblia hebraica et graeca. s.d.

73 Bible. Latine.

s.d.

Biblia sacra. Paris, 1651. 7 vol.

1745

Ex dono Casot, 1795 .

Castanet, ptre

Collège S.-Marie

74 Bible. A.T. Latine.

Libri Josue, Judicum, Ruth, Regum IV et Paralipomenon II. Cologne, 1639.

75 Bible. N.T. Latine.

Miss. Nov. Fr. 1745

Noveum Testamentum. Utrecht, 1675.

$1720 / 1745$ 
76 Bible N.T. Française.

Le Nouveau Testament de Nostre-Seigneur Jésus-Christ trad. en français. Paris, 1704.

77 Bible. Latine.

Reçu du P. Casot, juin 1795

L. J. Desjardins (*)

Biblia magna comment... J. Gagnaei, Guil. Estii, Emm. Sa, J. Menochii et Jacobi Tirini, Paris, 1643. 5 vol.

78 La Bibliothèque des poètes latins et français. Paris, 1731 s.d./1745

79 Binet, Etienne, s.j., 1569-1639.

Le riche sauvé par la porte dorée du ciel. Paris, 1627

80 Bion, Nicolas, 1652-1733.

s.d./1745

Traité de la construction et des principaux usages des instruments de mathématique... Paris, 1716

81 Biroat, Jacques. 1745

Frs. LeGuerne, 1753

Panégyriques des saints. Paris, 1669.

$1720 / 1745$

Hôp. Gén.

82 Blondel Saint-Aubin, Guillaume.

Trigonométrie géométrique, astronomique et maritime ... Hâvre-de-Grâce, 1718.

83 Boerhaave, Herman, 1668-1738.

1745

Institutions de médecine ... Paris, 1743

84 Boerhaave, Herman

Institutions de médecine ... Paris, 1740.

85 Id.

Tractatus de viribus medicamentorum. Paris, 1723.

86 Boileau

Oeuvres. 3 vol.

87 Boissieu, Antoine, s.j.

La vie du vénérable Frère Alphonse Rodriguez, s.j. Lyon, 1688.

88 Bonacina, Martin, -1631.

Opera omnia. Paris, 1632. 2 vol.

89 Id.

Id. Opera omnia. Paris, 1633

$1720 / 1745$

s.d./1745

s.d./1745

$\left(^{*}\right)$ Louis-Joseph Desjardins, 1766-1848, prêtre français, émigré à Québec en 1794, à la Révolution française. Chapelain de l'Hôtel-Dieu en 1807, et supérieur des Ursulines, en 1825.

Prix d'encouragement à Armand Dugast/Carleton 17 juin 1799 L. J. Desjardins Adolphe Dubast, 1837 Collège Sainte-Marie 
90 Bonaventure, $\mathrm{S}$.

Opuscula. Lyon, 1619. 2 vol.

Miss. Nov. Fr. 1745

91 Id.

Summa theologica. Lyon, 1616.

Miss. Nov. Fr. 1745

92 Borromée, Charles, saint, 1538-1584. Acta ecclesiæ Mediolanensis ... Paris, 1643.

93 Bossuet

s.d. $/ 1745$

Mandement et instruction pastorale ... sur le jansénisme. Paris, 1710 .

s.d. $/ 1745$

94 Bouchel, Laurent, 1559-1629.

La bibliothèque ou trésor du droit français . . . Paris, 1671. 3 vol.

1745

95 Bouguer, Pierre, 1698-1758.

Traité complet de la navigation ... Paris.

96 Bonhours, Dominique, s.j., 1628-1702.

1745

La vie de saint Ignace, fondateur de la compagnie de Jésus. Paris, 1679.

1745

Hôp. Gén.

97 Bourdaloue

Conscienes ... habitæ ... par adventum ... coram rege ... Angers, 1713-1715. 2 vol.

$1720 / 1745$

98 Id.

Concionum ... in quadragesimam ... Angers, 1711.

99 Bourdaloue

100 Id. Pensées

$1714,1720,1745$

C

Pensées... sur divers sujets de religion et de morale. $4 \mathrm{e}$ éd. Paris, 1740.

$$
1745
$$

Ménagerie

101 Id

Id. Sermons pour les dimanches. Paris, 1716.

Hôp. Gén.

1745

Poulin de Courval, ptre 1782

Hôp. Gén.

102 Bournonville, M. Valentin de. Missa quatuor vocum ... Paris, 1646.

103 Boutaud, P., s.j.

Méthode pour converser avec Dieu. Paris, 1685

s.d.

104 Bretonneau, François, s.j., 1660-1741.

Sermons. 3 vol.

$1703 / 1745$

C.

105 Breviarium romanum. Paris, 1670

Hôp. Gén.

106 Breviaire. 2 vol.

C. 
107 Breviarium romanum.

C.

108 Briet, Philippe, s.j., 1601-1668.

Annales mundi seu chronicon universale. Paris, 1662-1663. 6 vol.

109 Brunet, Claude.

s.d./1745

110 Id

Le progrès de la médecine ... pour l'année 1697. Paris, 1698

Id. ... pour l'année 1709. Paris, 1709.

1745

111 Buffier, Claude, s.j., 1661-1737.

1745

Cours de sciences ... Paris, 1732.

(Donum authoris ...)

1745

Hôp. Gér.

112 Busenbaum, Hermann, s.j. Medulla theologiæ moralis. 1698

B

113 Bussières, Jean de, s.j., 1607-1679.

Conciones ... Lyon, 1665. 2 vol.

114 Cabassut, Jean, 1604-1685.

$1720 / 1745$

Notitia conciliorum sanctæ Ecclesiæ . . . Lyon, 1670

115 Cachupin, François.

s.d./1745

La vie du Vén. P. Louis Dupont... Paris, 1663.

116 Caignet, Antoine, -1669.

L'année pastorale ... Paris, 1663-69. 2 vol.

117 Camaret, Louis,

La morale de Jésus-Christ . . L Lyon, 1693.

118 Cappel, Louis, 1585-1658.

Critica sacr. Paris, 1650.

119 Le caractère de la véritable et fausse piété.

Miss. Can.

1745

$1666 / 1745$

1745

1745

120 Cassien, Jean.

Les conférences de Cassien trad. en français ... Lyon, 1682. (Ex dono March. Brisaux Denonville)

121 Castelli, Bartholomée.

s.d. $/ 1745$

Lexicon medicum græco-latinum ... Rouen, 1665

122 Castillon, André, s.j.

Sermons pour les dimanches et festes de l'Advent. Paris, 1672

s.d. $/ 1745$

Hôp. Gén.

123 Le catéchisme du Concile de Trente. Paris, 1686.

1745

124 Catéchisme en images.

B

125 Catrou, François et Rouillé, s.j.

Histoire romaine. Paris, 1725. 21 vol. 
126 Caufapé, Anicet.

Nouvelle explication des fièvres et de la gangrène ... Avignon, 1687.

127 Id. $1720 / 1745$

Nouvelle explication des fièvres. Paris, 1697.

128 Id.

Réflexions singulières sur le fréquent usage de la saignée. Toulouse, 1696

129 Caussin, Nicolas, s.j., 1583-1651.

Regnum Dei seu dissertationes in libros regum . . Paris, 1650

130 Chahu, Philippe, s.j.

$$
\text { s.d./1745 }
$$

Le secret de la prédestination sur le petit nombre des élus... Paris, 1659.

131 Chaillou, Jacques.

s.d./1745

Recherches de l'origine et du mouvement du sang, du cœur... Paris, 1699.

132 Charpy de Sainte-Croix, Louis.

Catéchisme eucharistique. Paris, 1668.

133 Chastenet, Léonard.

s.d./1745

La vie de Mgr Alain de Solminihac ... Caors, 1663.

134 Cheminais de Montaigu, Timoléon, s.j.

Sermons. 1690.

Hôp. Gén.

135 Id.

Sermons. 1702.

136 Chevalier

$\mathrm{C}$

Réflexions critiques sur le traité de l'usage des différentes saignées ... Paris, 1730

137 Chirinos, Ferdinand de Salazar, s.j.

Hôp. Gén.

Expositio in proverbia Salomonis. Lyon, 1637. 2 vol.

s.d./1745

138 Le chrétien en solitude.

C

139 Cicéron

De oratore. Paris, 1640.

140 Id.

Entretiens. 2 vol.

s.d./1745

C

141 Id.

Epistres familières. Paris, 1666.

142 Id.

Lucubrationes in Ciceronis orationes. Bale, 1553.

143 Id.

Opera. 1576. 2 vol. 
144 Id.

De la vieillesse. Paris, 1698.

145 Col de Villars, Elie.

s.d.

Cours de chirurgie. Paris, 1738-1741. 1745

146 Comitin, César-Jean-Baptiste, s.j.

Hôp. Gén.

Le commencement et la perfection de la sagesse ... Paris, 1732

s.d./1745

147 Conduite chrétienne dans les états différents de la vie ...Paris, 1706 $1719 / 1745$

148 La conduite de saint Ignace

149 Conférences sur l'usure et la restitution.

C

150 La connaissance des temps ... pour l'année 1694. Paris, 1694.

151 De la connaissance et de l'amour.

152 Constant de Rebecque, I. de m.

L'apothicaire français charitable ... Lyon, 1683.

$1720 / 1745$

Ex dono R.P. Casot 1796 Desjardins

153 Id

Hôp. Gén.

d.

Le médecin français charitable ... Lyon, $1683 . \quad 1720 / 1745$

Desjardins

Hôp. Gén.

154 Cornelius Nepos.

Liber de vita excellentium virorum. Paris, 1569.

$$
\text { s.d./1799 }
$$

(Ex libris Joseph-Levasseur Borgia, 1799) (*)

155 Corneille à La Pierre, Corn.

Commentarii ... Josue, Judicum, Ruth, iv libros regum ... Anvers, 1653.

156 Id.

s.d./1745

Commentarii in iv Evangelia ... Lyon, 1649.

157 Id.

Id. Commentarii in Josue, Judices et Ruth ... Paris, 1642.

(Ex dono Seb. Cramoisy).

Miss. Nov. Fr.

s.d. $/ 1745$

158 Id.

In omnes D. Pauli commentaria ... Lyon, 1644.

159 Id.

s.d./1745

Ecclesiasticus ... Paris, 1642.

160 Id.

Salomon sive commentarius in Proverbia ... Paris, 1635.

Miss. Nov. Fr.

1745

$\left(^{*}\right)$ Avocat et homme politique, 1773-1839. 
161 Corpus juris civilis, 1559.

(Ex dono D. Jacobi Duchesneau, prætoris).

162 Corpus omnium voterum pœtarum, Genève, 1640 .

163 Cotterau Duclos, Samuel.

Observations sur les eaux minérales de France... Paris, 1675.

164 Courtot, François.

La vie de saint Pierre d'Alcantara ... Paris, 1670.

165 Crasset, Jean, s.j., 1618-1692.

s.d. $/ 1745$

Instructions spirituelles pour la guérison et la consolation des malades ... Paris, 1680. 2 vol.

(Ex dono Denonville).

s.d./1745

1 Ex. double.

166 Cressolles, Louis, s.j. 1568-1634.

Anthologia sacra. Paris, 1632. 2 vol.

(Ex dono Seb. Cramoisy).

167 Croiset, Jean, s.j.

$1632 / 1745$

Exercices de piété pour tous les dimanches et fêtes de l'année... Paris, 1727.

168 Croll, Oswald.

Basilica chimica. Genève, 1643.

169 Id.

La Royale Chimie. 1623.

$1720 / 1745$

170 Dalechamp, Jacques.

1745

Histoire générale des plantes ... Lyon, 1643. 1720/1745

171 Danet, Pierre.

Hôp. Gén.

Magnum dictionarium latinum et gallicum. Lyon, 1737.

Hôp. Gén.

172 Daubenton, Guillaume, 1648-1723.

La vie du bienheureux Jean-François Régis, s.j., Lyon, 1717.

173 Dauchet.

1720

Invitation aux muses pour célébrer le nouveau régime. Paris, 1716.

174 David, Jean.

Hôp. Gén.

Des jugements canoniques des évêques. Paris, 1671.

175 Deschamps, Etienne, s.j., 1613-1701.

De hæresi janseniana ... Paris, 1654.

1745

Miss. Nov. Fr. s.d. $/ 1745$

176 Deidier, Antoine.

Dissertation médicinale sur les maladies vénériennes ... Paris, 1735.

177 Id. 1745

Traité des tumeurs contre nature. Paris, 1732. 
178 Delidel, Claude, s.j.

La théologie des saints. Paris, 1668.

1745

Hôp. Gén.

179 De l'Orme, Philibert, 1518-1577.

Le premier tome de l'architecture. Paris, 1568.

180 Del Rio, Martin-Antoine, s.j., 1551-1608.

In Canticum Canticorum Salomonis commentarii... Ingolstadt, 1604.

181 Denis, Jean-Baptiste.

Mémoires, conférences et observations sur les arts et les sciences... Paris, 1682.

182 De Saint-André.

$1720 / 1745$

Réflexions nouvelles sur les causes des maladies. Paris, 1688.

1745

183 De Saulx.

Hôp. Gén.

Nouvelles découvertes concernant la santé et les maladies les plus fréquentes ... Paris, 1727.

184 Deschampneufs, Pierre.

1745

Psalmi Davidici et sacra cantica ... Paris, 1648.

185 De Serpa, Antoine.

s.d./1745

Eucharisticæ chronologiæ ... Paris, 1648.

186 Deshaies-Gendron, Claude.

s.d./1745

Recherches sur la nature et la guérison des cancers ... Paris, 1701.

187 Desmarets, I.

$1720 / 1745$

Les délices de l'esprit. Dialogues dédiés aux beaux esprits du monde. Paris, 1659.

Miss. Nov. Fr.

s.d. $/ 1745$

Hôp. Gén.

188 Desse.

Traité de la véritable connaissance des fièvres. Paris, 1691. Pharmacopée Coll. Jés.

189 Devaux, Léon, 1649-1729.

$1701 / 1745$

Hôp. Gén.

L'art de faire les rapports en chirurgie, Paris, 1703.

190 La dévotion à Jésus-Christ.

1720

191 Dictionnaire de Trévoux. 7 vol. C

192 Dictionnaire économique. 2 vol. C

193 Dictionnaire géographique. C

194 Digestum vetus seu pandectarum juris civilis. Paris, 1559, 3 vol.

195 Dodard, Denis, 1634-1707.

Mémoires pour servir à l'histoire des plantes. Paris, 1679. 
196 Dodœens, Rembert, 1518-1585.

Florum ... historia. Anvers, 1568.

Miss. Nov. Franc. 1632 1745

197 Id. Ex-libris Dr Jos. Parent

\section{d.}

Historia frumentorum, leguminum, palustrium et aquatilium ... Anvers, 1569.

Miss. Nov. Franc. 1632 1745

198 Id.

Purgantium aliarumque eo facientium ... historiæ ... Anvers, 1574.

Miss. Nov. Franc. 1632

1745

199 D'Orléans, Pierre-Joseph, s.j., 1644-1694.

La vie du P. Matthieu Ricci, s.j. Paris, 1693.

200 Dozenne, Pierre, s.j.

$1720 / 1745$

La morale de Jésus-Christ. Paris, 1686.

s.d./1745

Hôp. Gén.

201 Drexel, Jérémie, s.j., 1581-1638.

Cœlum beatorum civitas ... Anvers, 1636.

202 Id.

1745

Infernus damnatorum carcer ... Cologne, 1632. Miss. Hur., s.d. 1745

Hôp. Gén.

203 Dubé, $M$.

La médecine abrégée en faveur des pauvres. Paris, 1692.

Apothicairerie Hơp. Gén.

204 Dubois, P. V.

Apothicairerie Hop. Gen.

Nouveau traité des scrofules ou tumeurs froides, des cancers et loupes. Paris, 1726.

Hôp. Gén.

205 Dubois, P.-Violette.

Suite des maladies chroniques et aigues. Paris, 1727.

206 Dubosc

1745

Le triomphe de saint Augustin et la délivrance de sa doctrine. Où l'on voit la condamnation des cinq propositions des Jansénistes. Paris, 1652.

s.d./1745

Ranvoyzé 1797

207 DuCygne, Martin, s.j.

Ciceronis orationum analysis ... Paris, 1704.

208 DuFay, s.j.

s.d./1745

Sermons pour le caresme. Lyon, 1738. 2 vol. s.d./1745

209 Duhau, Laurent, 1656-1726.

Méditations. 1694.

Hôp. Gén.

C

210 DuLaurens, André, 1558-1609.

Oeuvres. Paris, 1646. 2 vol.

211 Id.

Toutes les œuvres. Rouen, 1661.

s.d. 
212 DuMay, Louis, $\quad-1681$.

Le prudent voyageur contenant la description de l'Asie, l'Afrique et de l'A mérique ... Genève, 1681. 3 vol.

213 Dumoulin, M.

Nouveau traité du rhumatisme et des vapeurs. Paris, 1703.

214 Duncan, Daniel, 1649-1735.

Apothicairerie Hôp. Gén.

Explication nouvelle et méchanique des actions du cerveau ... des fonctions de l'âme... les parties du cerveau ... Paris, 1678.

215 DuPerron, Jacques, card., 1556-1658. 1720/1745

216 DuPont, Louis, s.j. Traité du sainct sacrement de l'Eucharistie... Paris, 1622. Méditations sur les mystères de la Foy ... Paris, 1683.

217 Id.

1745 Méditations sur les mystères de la Foy. Paris, 1684. 1745

218 Id. Méditations.

219 Edinbourg, Société d'. Essais et observations de médecine... Paris, 1740-43. 5 vol.

220 Entretiens de M. le Commandeur de XXX. 1745

221 Les entretiens physiques. C

222 Epistolæ præpositorum generalium ad patres et fratres Soc. Jesu.

223 Est, Guillaum Hessels van. In omnes beati Pauli ... epistolas commentaria. Paris, 1659. 2 vol. C Hôp. Gén.

224 Etablissement de la foi. s.d./1745

225 Ettmüller, Michel, 1644-1683. Nouveaux instituts de médecine. Lyon, 1693.

226 Id.

C

227 Id. Pratique spéciale de médecine ... Lyon, 1698. Pratique générale de médecine. Lyon, 1699. 2 vol.

228 Euclide.

228 Elementorum libri xv. Paris, 1516.

229 Eudes, Jean, saint, 1601-1680. Le bon confesseur ... Paris, 1666.

230 Eusèbe, Pamphile. Præparatio evangelica. Paris, 1628. (Colleg. Paris. Soc. Jesu). 
231 Exercices de piété. 6 vol.

232 Extrait du rituel romain.

233 Fabricius, Jean-Albert, 1668-1736.

Codex apocryphus novi testamenti ... Hambourg, 1703. 2 vol.

234 Fénelon.

Instruction pastorale... sur la nécessité de rapporter toutes ses actions à Dieu. Paris, 1734.

235 Id.

Mandement au sujet d'un écrit ... Paris, 1730.

236 Filliucci, Vincenzio, s.j.

De christianis officiis et casibus conscientiæ... Lyon, 1622. 2 vol. (Domus prof. Paris.).

237 Fléchier.

Oraison funèbre de m ... Michel LeTellier. Paris, 1686.

238 Fouquet, Marie de Maupéon, 1590-1681.

Hôp. Gén.

Les remèdes charitables. Lyon, 1682.

239 Id

des remèdes charitables. Lyon, 1696.

1745

240 Fournier, George, s.j., 1595-1652.

Hydrographie contenant... toutes les parties de la navigation. Paris, 1643.

241 Id.

Hydrographie ... Paris, 1667.

242 François de Sales, S.

1745

243 Id

Introduction à la vie dévote.

1745

Oeuvres.

C

244 Freind, John, 1675-1728.

Histoire de la médecine ... Paris, 1728.

245 Fremart, Henri.

Missa quatuor vocum. Paris, 1642.

246 Frenicle, Epiphane.

Les mystères contenus dans les offices divins ... Paris, 1655.

247 Frey de Neuville, Pierre-Charles, s.j., 1692-1775.

s.d./1745

Sermons. 6 vol.

C

248 Fritach, Adam.

L'architecture militaire ... Leide, 1635.

249 G.

La mécanique du feu. Paris, 1713. 
250 Galliffet, Joseph, s.j.

L'excellence de la dévotion du Cœur adorable de Jésus-Christ. Nancy, 1745.

251 Gaillard.

H.d.

Oraison funèbre de . . A Adélaïde Savoy. Paris, 1712. 1720

252 Gallois, Antoine.

Hôp. Gén.

Oraison funèbre de ... Marie Thérèse d'Autriche. Paris, 1683.

$$
1720
$$

Hôp. Gén.

253 Garcia del Valle Calaguritano, Fr.

Evangelicus concionator et novi hominis institutio... Lyon, 1622. 2 vol.

254 Garengeot, René-Jacques Croissant de, 1688-1759.

s.d./1745

Traité des opérations de chirurgie. Paris, 1731. 3 vol.

255 Garnier, Pierre.

1745

Nouvelles formules de médecine latines et françaises pour le Grand Hôtel-Dieu de Lyon. Lyon, 1706.

256 Gautruche, Pierre, s.j., 1602-1681.

Hôp. Gén.

Philosophiæ ac mathematicæ ... institutio. Cadomi, 1665.

$$
\text { s.d./1745 }
$$

257 Id.

Ex libr. H. Verreau *
L'histoire sainte. Paris, 1702.
à l'usage de la classe.
s.d./1745
Hôp. Gén.

258 Gazette et Nouvelles ordinaires. 1679-1731. 45 vol.

259 Gimignano, Bernard de.

1720

Le antichita della citta di Roma. Vinegia, 1580.

(Ex libr. Colleg. Met. Soc. Jesu 1697).

260 Giroust, Jacques, s.j.

s.d.

Les faux prétextes du pécheur. Paris, 1700.

261 Id.

Sermons pour le caresme. Paris, 1704.

$1720 / 1745$

262 Godeau, Antoine, 1605-1672.

Histoire de l'Eglise. Paris, 1672. 4 vol.

263 Gondon, Gilles.

L'imitateur de Jésus-Christ ou la vie du vénérable Antoine-Yvan. Paris, 1662.

264 Gondon, James Lesmore, 1553-1641.

s.d./1745

Theologia moralis universa. Paris, 1634.

(Ex dono Seb. Cramoisy).

Miss. Nov. Fr.

s.d./1745

* Abbé Hospice-Anthelme, éducateur et historien (1828-1901). 
265 Gouet, Louis-Léger de.

La véritable chirurgie ... Rouen, 1716.

266 Gouttard, M.

Traité des eaux minérales d'Abbecourt... Paris, 1718.

267 Gr., Sr.

La conduite de l'homme chrestien ... Paris, 1679.

268 Graaf, Régnier de, 1641-1673.

Traité de la nature et de l'usage du suc pancréatique. Paris, 1666.

269 Grandamicus, Jacques, 1588-1672.

Chronologia christiana ... Paris, 1668. 3 vol.

270 Id.

De die supremo et natali Christi quæstio evangelica. Angers, 1661.

271 Grenade, Louis. s.d./1745 Catéchisme.

272 Id. 1811.

\section{Grisel, Jean, 1601-1657.}
s.d./1745
La vie de Dom Barthelemy ... Paris, 1664.
Hôp. Gén.

Le mystère de l'homme-Dieu. Paris, 1654.

274 Grotius, Hugo, 1583-1645.

s.d. $/ 1745$

De iure belli ac pacis libri tres ... Amsterdam, 1631.

275 De la guérison des fièvres par le quinquina ... Lyon, 1681.

276 Guisard, Pierre.

$1720 / 1745$

Dissertation pratique... sur les maux vénériens ... Paris, 1743.

277 Guy de Chauliac. 1745

Le maistre en chirurgie ... Paris, 1704.

278 Guyard, M.

$1720 / 1745$

De l'usage de la fréquente saignée dans les fièvres . . Paris, 1702.

279 Guybert, Philebert.

Le médecin charitable ... Paris, 1641.

280 Id.

$$
\text { Le medecin charitable... Paris, } 1641 \text { s.d./1745 }
$$

Toutes les œuvres charitables ... Paris, 1648.

281 Guyet, Charles.

Heortologia, sive de festis propriis ... Paris, 1657.

282 Guyon, Louis de, sieur de la Manche. 
283 Hacquenot, Henri, 1687-1775.

Recueil alphabétique de pronostics dangereux et mortels . . . Paris, 1736.

284 Hecquet, Philippe, 1661-1737.

Miss. Soc. Jes.

Le brigandage de la chirurgie ... Utrecht, 1738. 3 vol.

285 Id.

Hôp. Gén.

Traité des dispenses du carême... dissertations... sur les macreuses ... sur le tabac. Paris, 1710.

286 Henrion, Denis, -1640.

Mémoires mathématiques ... Paris, 1623.

287 Id.

1745

Mémoires mathématiques ... Paris, 1627.

288 Id.

s.d./1745

L'usage du mécomètre... Paris, 1630.

289 Hincmar, arch. de Reims.

Opera. Paris, 1645. 2 vol.

1745

Miss. Nov. Fr.

s.d./1745

290 Hippocrate.

Prænotiones. Paris, 1621.

291 Id.

1745

Les aphorismes ... Paris, 1634.

292 Id.

Aphorismes. Lyon, 1668.

1745

$1720 / 1745$

293 Histoire de France. 12 vol.

C

294 Histoire générale des drogues, des plantes, des animaux ... Paris, 1694.

295 Histoire générale des plantes ... Lyon, 1653.

Hôp. Gén.

296 1720/1745

296 Histoire journalière de ce qui se passe de plus considérable en Europe. La Haye, 1695-1701.

297 Histoire naturelle du cacao et du sucre .. Paris, 1719.

1720

298 Histoire sainte. 2 vol.

1745

299 Hoffmann, Friedrich, 1660-1742.

La médecine raisonnée... Paris, 1739. 2 vol.

300 Horace.

Carmina ... Tours, 1688.

301 Id.

Carmina ... Paris, 1697.

s.d./1745

$1720 / 1745$

302 Id.

Odes et satyres. Paris, 1670. 
303 Id.

Oeuvres. Paris, 1709. 10 vol.

s.d. $/ 1745$

304 Id.

Opera. 1653.

$1720 / 1745$

305 Id

Oeuvres. Paris, 1713.

s.d./1745

306 Hossch, Sidor, s.j., 1596-1653.

Elegiarum libri sex. Lyon, 1688.

307 Hoste, Paul, s.j., 1652-1700.

$1720 / 1745$

L'art des armées navales ... Lyon, 1697.

308 Id

Traité de la construction des vaisseaux ... Lyon, 1697.

309 Houdar de la Motte.

s.d.

Eloge funèbre de Louis le Grand ... Paris, 1716.

310 Houdry, Vincent.

Hôp. Gén.

La bibliothèque des prédicateurs . . . Lyon, 1712.

22 vol. (Ex dono authoris an. 1713

Ne domo affertur Houdry)

311 Hovel, Nicolas.

Traité de la thériaque et mithridate ... Paris, 1573.

Ad usum Pharmacopeiae Coll. Jes.

312 Ignace de Loyola, S., 1491-1556.

La conduite de saint Ignace . . . Bruxelles, 1661.

313 Instructiones ad provinciales et superiores Societatis . .

s.d./1745

Anvers, 1635.

314 Institutions au droit françois, 2 vol.

315 Instructions générales en forme de catéchisme ... Paris, 1702.

316 Justin, S.

Tou agiou Ioustinou sumgramata. Paris, 1551. 1720/1745

317 Ivo Carnotensis, ep.

Opera omnia. Paris, 1647. 2 vol.

318 Izqvierdo, Sebartien, s.j.

s.d./1745

Pharus scientiarum ... Aristotellis organon. Lyon, 1659.

319 Jacobus a $\mathbf{S}$. Michaele

s.d./1745

Sacrorum bibliorum idea ... Lyon, 1675. 2 vol.

320 Jansen, Corneille.

1745

Commentariorum in S. Concordiam . . epitone. Douai, 1593, (Dom. prob. paris. Soc. Jes.)

s.d. $/ 1745$

(*) Mgr Joseph-Octave Plessis, archevêque de Québec, 1763-1825. 
321 Id.

Tetrateuchus sive commentarius in s. J.C. evangelia. Lyon, 1676.

322 Jean Chrysostome, S.

Homiliæ.

323 Id.

$1720 / 1745$

Opera. Bâle, 1530.

324 Jean Climaque, Saint.

s.d./1745

Opera. Paris, 1633.

(ex dono Seb. Cramoisy)

325 Jerome, Saint.

Lettres. Paris, 1704.2 vol. s.d./1745

326 Jesus-Maria.

Hôp. Gén.

327 Johnston, John.

Dendrographias sive historiæ naturalis de arboribus et fructibus . . . libri decem. Francfort-sur-le-Mein, 1662.

328 Journal de Médecine ... Paris, 1683.

s.d. $/ 1745$

329 Le Journal des saints. 2 vol.

1745

330 Journal des savants. 1671-1683.

$1679 / 1720$

331 Journal historique. 1700-1707.

1720

332 Journal historique des assemblées tenues en Sorbonne pour condamner les mémoires de la Chine, \&c. 1701

333 Le journal ou histoire du temps présent ... 1651-1652.

$1720 / 1745$

334 Jousse, Mathurin.

1745

Le secret d'architecture. La Flèche, 1642.

335 Jude

$1720 / 1745$

Instructions pour les régents. (ms.) Archives du Séminaire de Québec.

(m.s.) Archives du

336 Junie ou les sentiments romains. Paris, 1695.

Séminaire de Québec

337 Justinien

$1720 / 1745$

Institutiones. Amsterdam, 1664.

338 Justinien, Laurent.

Ortus deliciarum. Milan, 1515.

(Ex libris Jean Grolier)

339 Juvénal

s.d./1745

Satires. Paris, 1658.

s.d./1745

340 Juvénal

Satyræ. Paris, 1700.

(Note ms.: Pour le P. Dupuys, s.j.)

Ex-libris J.-J. Borgia, 1799

$1721 / 1745$ 
341 Labbé Philippe, s.j.

Grammaticæ græcæ pœticæ libri tres. Paris, 1689. 1720/1745

342 LaCerda, Jean-Louis de, 1560-1643.

Hôp. Gén.

Adversaria sacra opus varium ... Lyon, 1626.

343 LaChambre, Marin Cureau de, 1594-1669.

s.d./1745

Les charactères des passions. Paris, 1659.

344 LaCharrière, Joseph de.

s.d./1745

Traité des opérations de la chirurgie. Paris, 1693.

345 LaColombière, Claude de, s.j.

$1720 / 1745$

Sermons ... Lyon, 1697.

s.d./1745

Hôp. Gén.

346 LaFramboisière, Nicolas-Abraham de.

347 LaHire, Philippe de, 1640-1718.

Tables astronomiques ... Paris, 1735.

348 Lalemant, Jérosme, s.j.

Relation ... $1645 \& 1646$. Paris, 1647.

349 LaMettrie, Julien Offray de, 1709-1751.

Traité de la petite vérole ... Paris, 1740.

350 Lamy, Guillaume.

Discours anatomique ... Paris, 1685.

351 Id.

Dissertation sur l'antimoine. Paris, 1682.

352 Lamy, Honoré

Abrégé chirurgical ... Paris, 1642. s.d.

353 Languet de Gergy, Jean-Joseph de, 1677-1753.

Première instruction pastorale ... de la constitution Univenitus. Paris, 1718.

354 Lancé, De.

s.d./1745

Oraison funèbre de . . . Elizabeth d'Orléans ... Alençon, 1696.

355 Lansberghe, Philippe de Meulebeecke, 1561-1632.

1720

Commentationes in motum terrae ... Medelbourg, 1630.

356 Id.

Tabulae motuum coelestium ... Middelbourg, 1653.

357 Lanuza, Jerome-Baptiste.

Homeliae quadragesimales ... Anvers, 1649, 4 vol. Miss. Nov. Fr.

358 LaRue, Charles de, 1643-1725.

Panégyriques des saints. Paris, 1740. 
359 Id.

Sermons ... pour le Caresme ... Lyon, 1719. 2 vol. 1745

360 LaSalle, Jean-Pierre
Traité des maladies de la poitrine. Bordeaux, 1704.

Hôp. Gén.

360a LaSanté, Xavier de., s.j.

Musae rhetoricis ... Paris, 1732.

(Donné par l'auteur au P. Deslandes)

361 Lauret, F.-H.

Sylva allegoriarum totius sacrae scripturae. Paris, 1584.

(Ex dono Dom. Prof. Paris.)

362 LaVauguion, De.

s.d./1745

Traité complet des opérations de chirurgie. Paris, 1698.

363 LeBlanc, Thomas, 1599-1669.

$1720 / 1745$

Psalmorum davidicorum analysis. Lyon, 1665-69. 3 vol.

364 LeBossu, Simon, s.j.

s.d./1745

L'usage de la grâce. Paris, 1659. 3 vol.

365 LeBret, Henry.

s.d./1745

Histoire de l'ancien et du nouveau testament . . . Paris, 1684.

Miss. Can.

s.d. $/ 1745$

Hôp. Gén.

$366 \quad \begin{aligned} & \text { Lebrun } \\ & \text { Superstitions anciennes et modernes. Amsterdam, } 1733 .\end{aligned}$

367 Lecat, Claude-Nicolas, 1700-1768.

Hôp. Gén.

Traité des sens. Paris, 1742.

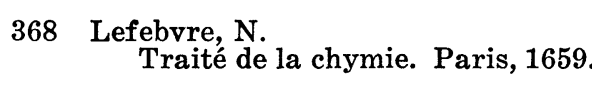

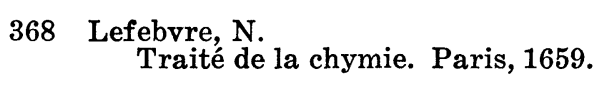

1745

369 LeFrançois, Alexandre.

Miss. Nov. Fr.

s.d./1745

Hôp. Gén.

Dissertation contre l'usage de soutenir des thèses en médecine . . . Paris, 1720.

370 Id.

Projet de réformation de la médecine. Paris, 1716.

371 Id.

$1720 / 1745$

Réflexions critiques sur la médecine ... Paris, 1714-15.

2 vol.

372 LeJay, Gabriel-François, s.j. 1657-1734.

$1720 / 1745$

Bibliotheca rhetorum ... Paris, 1725.

(Ex dono auctoris)

372a LeJeune, Jean, 1592-1672.

Le missionnaire de l'oratoire . . . Toulouse, 1667. 
373 LeJeune, Paul, s.j., 1591-1664.

Relation... 1633. Paris, 1634.

374 Id.

Relation ... 1638. Paris, 1638.

375 Id.

Relation ... 1656 \& 1657. Paris, 1658.

376 LeMarchant, Jacques, 1537-1609.

Hortus pastorum ... Rouen, 1652.

377 Id.

Hortus pastorum . . . Paris, 1661.

s.d.

$1720 / 1745$

s.d.

s.d.

Miss. Nov. Fr.

s.d./1745

378 LeMarchant, Jean.

L'encyclopédie sainte de la joy ... Rouen 1701. 1723/1745

379 LeMercier, François, s.j., $\quad-1685$.

Relation ... 1665 \& 1666 ... Paris, 1667.

- Ex double.

Hôp. Gén.

1720

380 Id.

Relation ... 1669- \& 1670. Paris, 1671.

381 Lemery, Nicolas, 1645-1715.

Traité universel des drogues simples. Paris, 1714.2 vol.

382 Lemonnier, L.

Hôtel-Dieu de Québec

Traité de la fistule de l'anus ou du fondement ... Paris, 1689.

1745

383 LeMoyne, Pierre, s.j., 1602-1672.

Apothicairerie-Hôp. Gén.

Saint Louys ou le héros chrétien, poëme héroïque. Paris, 1653.

(Relié aux armes de Jean Talon. Donné en prix au collège de Québec le 26 novembre 1671, à Noël Gaignon).

384 Id.

$1720 / 1745$

Les œuvres poétiques. Paris, 1672 . 1720/1745

385 Léon

Hôp. Gén.

Le confesseur justifié sur l'absolution différée . . . Paris, 1689.

386 Lessius, Leonard, s.j.

Opuscula. Anvers, 1626.

387 LeTellier, Michel, s.j., 1643-1719.

s.d. 1745

Miss. Nov. Fr.

Défense des nouveaux chrétiens . . . de la Chine, du Japon et des Indes contre deux livres ... La Morale des Jésuites et l'Esprit de M. Arnauld. Paris, 1687.

388 Lethieillier, Louis-Jean

Consultations de médecine . . . Paris, 1742.

s.d./1745

389 Lettre d'un docteur de $x x x$ à un Jésuite de la Province Gallo1745

Hôp. Gén. Belgique ... 1703.

- Ex. double. 
390 Lettre de MM. des Missions étrangères au Pape sur les idôlatries et les superstitions chinoises.

391 Lettres édifiantes et curieuses écrites des missions étrangères par quelques missionnaires de la Cie de Jésus. Paris, 1705.

$$
1720 / 1745
$$

392 LeVacher, Gilles, 1693-1760. Hôp. Gén.

Dissertation sur le cancer des mamelles. Bruxelles, 1740.

393 L'Hospital, Guillaume-François-Antoine de, 1661-1704.

Analyse des infiniment petits pour l'intelligence des lignes courbes. Paris, 1696.

394 Lingendes, Claude de, 1591-1660.

Conciones de sanctissimo eucharistiae sacramento. Paris, 1663.

395 Longus a Coriolano, Franciscus. Summa conciliorum ... Paris, $1639 . \quad$ Miss. Nov. Fr.

396 Lorin, Jean, s.j., 1559-1634. s.d. $/ 1745$

Commentarii in librum psalmorum. Lyon, 1616.

397 Id.

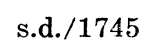

s.d. $/ 1745$

In acta apostolorum commentaria. Lyon, 1605.

(Domus Prof. Paris. soc. Jes.)

398 Losa, François.

La vie du bienheureux Grégoire Lopez. Paris, 1674.

399 Louis, Daniel.

Traité du bon choix des médicaments . . Lyon, 1710. 2 vol.

400 Lucrèce

De la nature des choses. Paris, 1685 .

401 Lugo, Jean de, 1583-1660.

Responsorum moralium libri sex. Lyon, 1660.

$402 \mathrm{M} \times \mathrm{xx}$

Institution au droit françois. Paris, 1719.

403 M., F.

M., F. Poésies spirituelles. Paris, 1671.

404 Mabillon, Jean, 1632-1707.

Traité des études monastiques. Paris, 1691.

405 Machault, Jacques de, s.j., 1600-1680.

Le Thresor des grands biens de la Très-Sainte Eucharistie . . . Paris, 1661.

Etiquette: Printing Office Quebec

406 Maerat, Louis.

Ex libris: Adrien Samson, Quebec.

1720

1745

1745

s.d. $/ 1745$

1745

Disputationum in summam theologicam S. Thomas tomi III.

Paris, 1633, 3 vol.

(Domus Prof. Paris, Soc. Jes.) 
407 Maffei, Giovanni-Pietro, 1536-1603.

Historiarum indicarum libri xvi. Cologne, 1593.

408 Magini, Giovanni-Antonio, 1555-1617.

Miss. Nov. Fr.

Supplementum ephemeridum ac tabularum secundorum mobilium. Venise, 1614.

409 Maimbroug, Louis, s.j., 1610-1686.

Histoire de l'Arianisme ... Paris, 1686. 2 vol.

410 Id.

411 Id

Histoire de l'hérésie des iconoclastes . . . Paris, 1686.

Histoire de la décadence de l'empire après Charlemagne . . .

$1720 / 1745$

Paris, 1686.

412 Id

$1720 / 1745$

Histoire de la Ligue. Paris, 1686.

413 Id.

$1720 / 1745$

Histoire du Calvinisme. Paris, 1686.

414 Id.

$1720 / 1745$

Histoire du Grand Schisme d'Occident. Paris, 1686.

415 Id.

$1720 / 1745$

Histoire du Luthéranisme. Paris, 1686.

416 Id.

$1720 / 1745$

Histoire du schisme des Grecs. Paris, 1686. 417 Id.

Traité historique de l'établissement et des prérogatives de l'Eglise de Rome et des évêques. Paris, 1686.

418 Maitrejean, Antoine.

$1720 / 1745$

Traité des maladies de l'œil ... Troyes, 1707.

419 Le Maître italien.

1745

420 Maldonado, Juan, s.j., 1533-1583.

Commentarii in præcipuos sacræ scripturæ libros veteris testamenti. Paris, 1614.

421 Manfred, Eustache, 1674-1739.

Miss. Nov. Fr.

$1644 / 1745$

Novissimæ ephemerides motuum coelestium . . Bologne, 1725. 2 vol.

422 Manni, Jean-Baptiste, s.j.

1745

Les quatre éternitez. Paris, 1687.

423 Manquest de la Motte, Guillaume, 1655-1737.

Resid. Marianap.

Traité complet de chirurgie... Paris, 1732. 4 vol. 1725 
424 Mantelio, P. F. Jo.

Speculum peccatorum ... sive admiranda S. Augustini conversio ... Anvers, 1637. s.d./1745 Abbé Verreau $(*)$ Hôp. Gén.

425 Marande, Sieur de. s.d. $/ 1745$

426 Mariana, Juan de, s.j., 1536-1624.

La clef de saint Thomas ... Paris, 1668-69. 10 vol.

Histoire générale d'Espagne ... Paris, 1725.

(Don du traducteur)

(1er envoi perdu dans le naufrage du Chameau)

1745

427 Id.

Scholia in vetus et novum testamentum. Paris, 1620.

(Domus Prof. Paris.)

s.d. $/ 1745$

428 Marie de l'Incarnation

Retraites ... avec une exposition ... du Cantique des Cantiques. Paris, 1682.

429 Marolles, Claude de, s.j., 1712-1792.

s.d./1745

Hôp. Gén.

\section{Sermons. 2 vol.}

C

430 Martial

Epigrammata ... Paris, 1693.

431 Martin, M.

Traité de la phlébotomie et de l'artériotomie.

Paris, 1741.

432 Martin, Barthélemy.

Traité du lait ... Paris, 1706.

Traité du lait... Paris, 1706.

433 Martinon, Jean

$1720 / 1745$

Disputationes theologicae... Paris, 1643. 5 vol.

s.d./1745

434 Martyrologe romain.

C

435 Mascarenhas, Garcia de.

Tractatus de sacramentis in genera . . . Paris, 1656.

(Ex dono Cramoisy)

Coll. Keg. 7 jul. 1657

436 Maubet, $M$. 1745

Traité de la dyssenterie ... Paris, 1712.

437 Id

Traité des tumeurs et des obstructions. Paris, 1702. 1745

438 Maupertuis, Pierre-Louis-Moreau de, 1698-1759.

Hôp. Gén.

Discours sur les différentes figures des astres ...

Paris, 1742.

Abbé Hospice-Anthelme Verreau, éducateur et historien (1828- 
439 La médecine théologique ... Paris, 1733. 2 vol.

440 Mémoires du Levant. 8 vol.

441 Menage, Egide.

Poëmata. Paris, 1668.

442 Mendoza, François de.

Comment. in quatuor libros regum . . . Cologne, 1628-1632. 3 vol.

Miss. Nov. Fr. 1745

443 Menochio, Giovanni-Stefano, s.j., 1576-1655. De republica hebraeorum ... Paris, 1648.

Miss. Nov. Fr.

444 Le Mercure Galant. 1679, 1685-1688, 1702, 1703, 1708. 1745

445 Mery, Jean, 1645-1722.

Observations sur la manière de tailler dans les deux sexes ... Paris, 1700.

$1720 / 1745$

446 Méthode pour converser avec Dieu.

C

447 Meuve, De. Dictionnaire pharmaceutique ... Paris.

1745

Hôtel-Dieu de Québec

448 Mezzavacca, Flaminio, -1704. Otio sive ephemerides Felsineae . . ab anno 1701 ad 1720. Bologne, 1701. 2 vol.

449 Michallet Les principes de la géographie ... Paris, 1690.

450 Michow, Justyn de, o.p.

Discursus praedicabiles super litanias B. Mariae Virginis ... Paris, 1642. 2 vol.

Miss Nov. Fr. 1745

451 Minot, Jacques.

De la nature et des causes de la fièvre avec . . expériences sur le quinquina. Paris, 1684.

452 Missale romanum ... Reims, 1628.

$1720 / 1745$

453 Missel. (Au Couvent de Pierreville)

$1720 / 1745$

454 Missel romain. 1698.

B

455 Monardes, Nicolas. $-\mathbf{1 5 7 8 .}$

De simplicibus medicamentis in Occidentali India delatis . . Anvers, 1574.

Miss. Nov. Fr. 1632

1745

456 Montanus, Ben Arias.

Novum testamentum graecum. Anvers, 1584.

457 Montereul, Bernardin, s.j.

s.d./1745

La vie du Sauveur du monde, Jésus-Christ, tirée des quatre évangélistes ... Paris, 1651. 
458 Moraines, Antoine.

Anti-Jansenius ... Paris, 1652.

459 Moreau, Jacques.

s.d./1745

De la véritable connaissance des fièvres. Paris, 1685 .

460 Mornac, Antoine, 1554-1619.

Observationes in xxiv libros digestorum . . . Paris, 1646.

(Ex dono D. Jacobi Duchesneau) (*)

461 Mornac, Philippe de, 1549-1623.

De la vérité de la religion chrétienne. 1585 .

(Ex dono Lauzon de Charny) (**)

462 Morton, Richard, 1635-1698

s.d./1745

Opera medica ... Lyon, 1697.

463 Mothier, Simon, s.j.

Le martyrologe romain. Paris, 1705.

464 (Mystères du Père à deux étoiles)

C

465 N., J.

Observations curieuses sur . . . la médecine et la chirurgie . . . Brest, 17.

466 Nepveu, François, s.j., 1639-1708.

1745

Retraite selon l'esprit \& la méthode de saint Ignace . . Paris, 1687.

467 Id.

s.d. $/ 1745$ Retraite selon l'esprit et la méthode de saint Ignace $\underset{17 \dot{45}}{\text { 1691. }}$ Paris,

Hôp. Gén.

468 Nicéron, Jean-François, 1613-1646.

La perspective curieuse ... Paris, 1638.

469 Nicole, Pierre, 1625-1695.

s.d./1745

La conférence du diable avec Luther contre le saint sacrifice de la messe. Paris, 1673.

(Ex dono D. Jacobi Duchesneau in Nov. Fr. praetoris)

470 Nuremberg, Jean-Eusèbe, s.j., 1595-1658.

De adoratione in spiritu et veritate.

s.d./1745

Anvers, 1631.

Miss. Nov. Fr.

471 Id. 1745

Doctrina ascetica. Lyon, 1643.

Miss. Nov. Fr. 1745

472 Noël, Jérôme, s.j., 1507-1580.

Adnotationes et meditationes in evangelia ... Anvers, 1594.

Miss. Huron, S.J.

s.d./1745

(*) Intendant de la Nouvelle-France de 1675 à 1682.

(**) Vicaire général de la Nouvelle-France, 1660 à 1671. 
473 Id.

Evangelicae historiae imagines. Anvers, 1586. B

473a Id.

Id. Anvers, 1692.

B

474 Nostri saeculi ornamentum . . . Alexander Bertius. Rouen, 1646.

475 Novet, Jean, s.j., 1603-1680.

1745

Méditation et entretien sur le bon usage des indulgences. 1677.

476 Nouveau cours de chymie, suivant les principes de Newton et de Sthall ... Paris, 1723. 1745

477 Le Nouveau Mercure Galant. 1677, 1705.

1720

478 Le Nouveau Testament.

C

479 Nouveaux éléments de géométrie ... Paris, 1667.

480 Nouveaux mémoires des missions de la Compagnie de Jésus. 9 vol.

481 Nouveaux mémoires des missions de la Compagnie de Jésus. Paris, 1715. 2 vol.

482 Nouvelle chirurgie médicale.

1745

483 Novus apparatus graeco-latinus ... Paris, 1665. 2 vol.

484 Observations de médecine ... Paris, 1689.

C

$1720 / 1745$

Hôp. Gén.

485 Observations sur la saignée du pied, sur la purgation au commencement de la petite vérole ... Paris, 1724 .

486 Odespung de la Machinière, Louis, $\quad-1597$.

1745 Concilia novissima Galliae... Paris, 1646.

487 L'Office du Saint-Sacrement. Paris, 1659.

488 Offices à l'usage de la Société de Jésus.

s.d./1745

s.d./1745

489 Id.

Supplément.

490 Ollivier, L.

Traité des maladies des reins et de la vessie. Rouen, 1631.

491 Ordo administrandi sacramenta.

C 1720

492 Ordre nouveau . . . des tables des sinus, tangentes et sécantes. Lyon, 1628.

493 Orlandini, Niccolo, 1554-1606.

s.d.

Historia Societatis Jesus. Anvers, 1620. 6 vol. 
494 Orta, Garcia de, 16e s.

Aromatum et simplicium aliquot medicamentorum apud Indos nascentium historia ... Anvers, 1574. Miss Nov. Fr. 1632

495 Ovide

Metamorphoses. Amsterdam, 1659.

496 Id.

Id. 1699.

497 Id

Id. Rouen, 1736.

498 Pagan, Blaise-François, 1604-1665.

Les tables astronomiques ... Paris, 1681.

499 Id.

La théorie des planètes . . . Paris, 1657.

500 Panégyriques des saints.

501 Paradisus animae christianae.

$\mathrm{C}$

502 Paré, Ambroise, 1517-1590.

Les œuvres ... Lyon, 1664 .

$1720 / 1745$

503 Parochiale ... ecclesiae Rothomagensis. Rouen, 1640. Miss. Nov. Fr.

504 Parsons, James, 1705-1770.

Description de la vessie urinaire de l'homme . . Paris, 1743.

505 Petau, Denis, s.j., 1583-1652.

1743

Theologica domata. Paris, 1644. 5 vol.

506 Petit, Jean-Louis, 1674-1750.

s.d./1745

Traité des maladies des os. Paris, 1723. 2 vol.

507 Pexenfelder, Michel, s.j.

Florus biblicus ... Strasbourg, 1672.

1745

508 Phèdre

Fables. Chalons, 1685.

509 Philippeaux, Jean, s.j.

Oseas ... commentariis illustratus. Paris, 1636. (Ex. dono Seb. Cramoisy)

Miss Nov. Fr.

s.d. $/ 1745$

510 Pierre de Saint-Joseph

Idea theologicae moralis. Paris, 1645.

511 Platel, Jacques, 1608-1681

Synopsis cursus theologici . . . Douai, 1678.

5 vol.

Miss. Canad.

s.d. $/ 1745$

$1720 / 1745$

512 Plaute

$1720 / 1745$

Comédies. Paris, 1683, 3 vol. 
513 Pline le jeune.

Lettres. Paris, 1700.

(Pour le P. Dupuy, note ms.)

s.d./1745

514 Pline

1585. (Livre donné en prix par J. Duchesneau, intendant, 1675-1682.)

515 Poire, François, s.j.

La triple couronne de la bienheureuse Vierge Mère de Dieu. Paris, 1639.

Miss Nov. Fr. 1745

516 Polan, Pierre Soave.

Histoire du Concile de Trente, Troyes, 1655.

517 Pomet, Pierre, 1658-1699.

s.d. $/ 1745$

Droguier curieux. Paris, 1709.

518 Id.

Histoire générale des drogues. Paris, 1694.

519 Pontas, Jean.

Dictionnaire des cas de conscience. 1741. 3 vol. C

520 Pontifical romain.

C

521 Porée, Charles, s.j., 1675-1741.

Oraison funèbre de Louis le Grand. Paris, 1716.

522 Pratique facile pour élever l'âme.

$\mathrm{C}$

523 Pratiques de dévotion pour honorer la Sainte Vierge. Paris, 1670. s.d./1745

524 Pratiques de piété.

C

525 Pratiques des cérémonies de la :Sainte Messe.

C

526 Processionale ecclesiae Rothomagensis. Rouen, 1645.

527 Le progrès de la vie spirituelle.

C

528 Prosodia latina. Lyon, 1667.

529 Quen, Jean de, s.j., 1603-1659.

Relation ... 1655 \& 1656. Paris, 1657.

530 Quesnay, François, 1694-1774.

L'art de guérir par la saignée. Paris, 1736.

531 Id.

Essai physique sur l'oeconomie animale. Paris, 1736.

532 Raemond, Florimond de, 1540-1602.

L'Anti-Christ et l'antipapesse. Paris, 1599.

533 Ragueneau, Paul, s.j., 1608-1680. 
534 Ragueneau, Paul, s.j., 1608-1680.

La vie de la Mère Catherine de Saint-Augustin, religieuse hospitalière de la Miséricorde de Québec en la Nouvelle-France. Paris, 1671.

1745

535 Rapin, René, s.j., 1621-1687.

Collège Sainte-Marie

La perfection du christianisme . . . Paris, 1690.

536 Raynier, Timothée de.

1750

La société des zélateurs de la pureté et intégrité de la très Sainte Vierge .... Aix, 1662.

537 Raulin, Jean.

Opera, Anvers, 1612. 2 vol.

s.d. $/ 1745$

$$
\text { Opera, Anvers, 1612. } 2 \text { vol. }
$$

538 Réaumur, René-Antoine Ferchauld de, 1683-1757.

Miss. Nov. Fr.

L'art de convertir le fer forgé en acier, ... Paris, 1722. 1745

539 Recueil de douze homélies pour les dimanches et fêtes de l'année . . . Paris, 1707.

540 Recueil de sermons sur les évangiles du carême...

Hôp. Gén.

1745

\section{Bruxelles, 1706.}

1745

Hôp. Gén.

541 Recueil des mandements . . . pour l'acceptation de la constitution de N.S. Père le Pape Clément xiv ... Paris, 1715.

$1719 / 1745$

542 Recueil historique des bulles, constitutions, brefs, décrets . . . concernant les erreurs de ces deux derniers siècles . . Mons, 1710 . 1745

543 Réflexions sur les défauts d'autrui ... Paris, 1693.

1745

Hôp. Gén.

544 Réflexions sur l'usage de l'opium ... Paris, 1726.

1745

545 Reginauld, Valère, 1543-1623.

Praxis fori poenitentialis . . . Cologne, 1633.

s.d./1745

546 Règles de la Compagnie de Jésus. Paris, 1620.

$1720 / 1745$

$546 \mathrm{a}$ Id.

C

547 Règles de la Compagnie de Jésus. 1734.

548 Regnault, Noël, s.j., 1683-1762.

L'origine ancienne de la physique nouvelle.

Paris, 1734. 3 vol.

1745

Hôtel-Dieu de Québec 1812.

Collège Sainte-Marie

549 Relation... $1664 \& 1665.1666$.

1745

$1720 / 1745$

Collège Sainte-Marie

550 Relation... $1668 \& 1669$. Paris, 1670.

551 Relation contenant la suite et conclusion du Journal 1652-1683.

552 Relations véritables. 1695, 1696. Bruxelles. s.d./1745

1720 
553 Remarques sur l'abus des purgatifs et des amers. Paris, 1729.

554 Renou, Jean de.

Les œuvres pharmaceutiques... Lyon, 1637.

555 Retraite de saint Ignace.

Miss. Nov. Franciæ

C

556 Reyneau, Charles-René, 1656-1728.

La science du calcul des grandeurs en général... Paris, 1714. 2 vol.

557 Ricci, Bartholomeo, 1490-1569.

Monotessoron evangelicum ... Poitiers, 1621.

558 Richelieu, Armand-Jean du Plessis, card. de.

Traité de la perfection du chrestien ... Paris, 1646.

559 Riolan, Jean, 1577-1657.

Chirurgie ... Paris, 1669.

560 Rivard, Dominique-François, 1697-1778.

Elémens de géométrie... Paris, 1739.

561 Rivière, Lazare, 1589-1655.

La pratique de la médecine ... Lyon, 1682. 2 vol.

562 Rodriguez, Alphonse, s.j.

$1720 / 1745$

Pratique de la perfection chrestienne ... Paris, $1679 . \quad 1745$

562a Id.

Id.

563 Roger, Eugène, récollet.

La Terre Sainte, description ... Paris, $1664 . \quad$ s.d./1745

564 Rohault, Jacques, 1620-1675.

Hôp. Gén.

Traité de physique... Lyon, 1681. 2 vol.

$1720 / 1745$

565 Rossi, Joh.-Bapt.

Distinctiones ex commentariis S. Bonaventuræ ... Paris, 1646.

(Ex dono Seb. Cramoisy).

566 Rostagny, Jean de.

s.d. $/ 1745$

Traité de Primœrose sur les erreurs vulgaires de la médecine... Lyon, 1689.

567 Rosweyd, Heribert, 1569-1629.

$1720 / 1745$

Vitæ Patrum ... Lyon, 1617.

568 Rousseau, 1630-1696. (Le Capucin du Louvre).

s.d./1745

Secrets et remèdes éprouvez ... Paris, 1697.

De la Pharmacopée du Collège.

569 Rousseau, Gilbert.

$1720 / 1745$

Méditations pour toute l'année... Limoges, 1677. 
570 Roussel, Godefroy.

Les secrets... tant de pharmacie que de celuy de distillier... Paris, 1618.

571 Rouvière, $M$.

Réflexions sur la fermentation et sur la nature du feu. Paris, 1708.

572 Rozfeld, Jean-Bartholomée, 1551-1626.

$1720 / 1745$

Romanarum antiquitatum libri decem ... Bâle, 1583.

(Ex dono de la Galissonnière).

573 Ruscelli, Girolamo, pseud. Alexis Piémontois.

Les secrets du seigneur Alexis ... Rouen, 1662.

1720

574 Sa, Emmanuel, s.j.

Aphorismi confessariorum ... Lyon, 1712.

Miss. Nov. Fr.

Duplic. 1745

Collège Ste-Marie

575 Sacrosancti Concilii Tridentini canones et decreta ... Lyon, 1624.

576 Saint-Jure, Jean-Baptiste, s.j.

Miss. Nov. Fr.

L'homme spirituel ... Paris, 1646.

577 Saint-Martin de la Porte, Antoine.

s.d./1745

Les conduites de la grâce... Paris, 1645. 2 vol. Miss. Nov. Fr.

578 Saint-Yves, de. 1745

Nouveau traité des maladies des yeux. Paris, 1722.

579 Sales, S. François de.

1745

Hôp. Gén.

Les epistres spirituelles ... Paris, 1676. 2 vol.

580 Salluste.

1745

Conjuratio catillina et bellum jugurthinum opus... Paris, 1725.

581 Salvien. 1745

Nouvelle traduction des œuvres de Salvien... Paris, 1700. 2 vol.

Hôp. Gén.

581a Id. Autre ex.

582 Sanchez, Thomas, 1550-1610.

Disputationum de s. matrimonio libri tres... Anvers, 1614. 3 vol. (Colleg. Paris. dedit Miss. Canad.).

583 Sanroman, Michel de, s.j.

Expeditionum spiritualium libri tres. Lyon, 1644.

584 Saussoy, André du, 1589-1675. Martyrologium gallicanum ... Paris, 1637. 2 vol.

585 Id.

Panoplia episcopalis ... Paris, 1646.

(Ex dono S. \& G. Cramoisy). 
586 Id.
Panoplia clericalis ... Paris, 1649.
s.d./1745

587 Id.

Panoplia sacerdotalis ... Paris, 1653. s.d./1745

588 Schott, Gaspard, s.j., 1608-1666.

Cursus mathematicus ... Herbipoli, 1661 . 1745

589 Schroder, Jean.

Pharmacopeia medico-chymica ... Lyon, 1665. 1670/1720

590 Sebastien, Frère.

Christiana institutio virtutum et vitiorum. Paris, 1650.

591 Sénèque.

592 Sennert, Daniel, 1572-1637.

s.d./1745

Opera omnia. Lyon, 1661. 2 vol.

s.d./1745

593 Sermons sur divers sujets moraux.

C

594 Sermons sur les mystères.

C

595 Sermons sur tous les sujets de la morale chrétienne. Paris, 1702. 2 vol. $1720 / 1745$

Hôp. Gén.

596 Séville, I. de. Le compost manuel calendrier et almanach perpétuel... Rouen, 1595.

597 Silva, Jean-Baptiste, 1682-1748.

Traité sur l'usage des différentes saignées... Paris, 1727. 2 vol. 1745

598 Smet, Henri.

Hôp. Gén.

Prosodia. Rouen, 1662.

$1720 / 1745$

599 Smith, John.

Traité des vertus médicinales de l'eau commune... Paris, 1725.

1745

600 Solitude intérieure. Paris, 1678.

1745

600a Les souffrances de Notre-Seigneur Jésus-Christ. Paris, 1696.

Duplic. 1745

Collège :Sainte-Marie

600b Les souffrances de Jésus-Christ. 3 vol.

C

601 Spectacle de la nature. 2 vol.

C

602 Stevin, Simon, -1635.

Oeuvres mathématiques ... Leyde, 1634.

603 Suarez, François.

In IIIam partem D. Thomæ ... Moguntiæ, 1610. 2 vol. 1745 
604 Id.

De divina gratia. 3 vol.

605 Id.

In 2dæ D. Thomæ... Lyon, 1621. 2 vol.

1745

606 Id.

De religione. Lyon, 1625.

(Ex dono Colleg. Paris., 1641).

s.d./1745

607 Symphorien, Benoit C.

Hortorum libri triginta. Lyon, 1560.

608 Taberne, J. B., s.j.

Synopsis theologiæ practicæ ... Douai, 1698. 3 vol 1745

609 Tarde, Jean.

Les usages du quadrant à l'esguille aimantée ... Paris, 1621.

610 Tauvry, Daniel, 1669-1701.

1745

Nouvelle astronomie raisonnée ... Paris, 1690. 1723/1745

611 Terrasson, André, 1669-1723.

Sermons ... Paris, 1726. 4 vol. s.d.

612 Tencke, $\mathrm{H}$.

Hôp. Gén.

Formules de médecine tirées de la pharmacie galénique et chymique. Lyon, 1712.

613 Terence, Publ.

Comœediæ. Amsterdam, 1641.

Hôp. Gén.

613a Id.

Comœdiæ. Lyon, 1635.

1745

Bibliothèque de Mgr Hubert

1745

614 Tertre, Jacques du, o.p., 1610-1687.

Histoire générale des Antilles ... Paris, 1667. 2 vol.

615 Tertre, Du, s.j., 1677-1762.

s.d./1745

Réfutation d'un nouveau système de métaphysique proposé par le P. Malebranche... Paris, 1715. 3 vol.

616 Texier, s.j.

1745

Sermons pour tous les jours de caresme ... Paris, 1675

s.d.

Ex-libris C. Genest, 1794.

617 Theodorat.

Orationes decem. Paris, 1630 s.d./1745

618 Théologie française.

C

619 De la théorie de la manœuvre des vaisseaux. Paris, 1689. 1745

620 Thesaurus pœticus.

C

621 Thévenin, François.

Opérations de chirurgie. Paris, 1658. 
622 Thibaut, $P$

Cours de chymie. Paris, 1667.

$1720 / 1745$

623 Thomas a Kempis.

De imitatione Christi libri quatuor. Anvers, 1617.

(De l'ancienne bibliothèque des Jésuites, offert au T. R. P. Ohuzel, jésuite, M. Desjardins, Québec.)

624 Thomas d'Aquin, S.

Collège Sainte-Marie

Catena aurea ... Paris, 1637.

625 Id.

Opuscula. Paris, 1634.

626 Thomas d'Aquin, S.

Summa contra gentiles. Anvers, 1568.

Miss. Nov. Fr. s.d./1745

Miss. Nov. Fr. 1745

Miss. Nov. Fr. 1745

627 Thomas de Jésus.

Les souffrances de Jésus-Christ. Paris, 1685.

s.d.

628 Toledo, Francis de.

Hôp. Gén.

In Ioannis evangelium commentarii. Rome, 1588.

Miss. S. Fr. Xavierii

$1643 / 1745$

629 Tournefort, Joseph-Pitton de, 1656-1708.

Traité de la matière médicale ... Paris, 1717. 1720/1745

630 Tournemine. s.j.

Instruction pour les régents de la Compagnie.

(Ms.) (Note ms.: Ce manuscrit appartenait à la succession de Mgr l'Evêque de Capsa...).*

631 Traité de la mort de Dieu.

C

632 Traité de la nouvelle orthographe.

C

633 Traité de la peste... Paris, 1722.

Hôp. Gén.

634 Traité de la vérité de la religion chrétienne... Rotterdam, 1689. $1720 / 1745$

635 Traité du scorbut... LaRochelle, 1671.

1745

636 Tronçon, Jean.

Le droict français et coutume de la prévosté et vicomté de Paris ... Paris, 1652.

637 Turrien, Louis.

1745

Disputationes in secundam secundæ D. Thomæ... Lyon, 1617. 2 vol.

(Dom. Prof. Soc. Jesu. Paris.).

s.d./1745

638 Valens, François.

Concordia juris pontificii cum cæsareo ... Paris, 1654.

Miss. Nov. Franc.

1745

* Mgr Jean-François Hubert, 9ème évêque de Québec (1739-1797). 
639 Van den Sten, Cornelis Cornelissen, s.j., 1567-1637.

In ecclesiasten commentaria ... Paris, 1639.

(Ex dono S. Cramoisy).

Miss. Nov. Franc.

1745

640 Vanurius, Jacques, s.j., 1664-1739.

Prædium rusticum. Paris, 1707.

(Pour le p. DuPuys à Québec).

$1721 / 1745$

641 Varignon, Pierre, 1650-1722.

Projet d'une nouvelle méchanique ... Paris, 1687.

642 Vazquez, Gabriel.

Comment. ac disput. in Iam IIdæ S. Thomæ. Anvers, 1621.

(P. Hazeur dedit Colleg. Quebec).

1745

1745

643 Velasquez, Jean-Antoine, 1585-1669.

In psalmum Davidis centesimum commentarii ... Anvers, 1640.

(Domus prof. Paris.).

s.d. $/ 1745$

644 Id.

In epistolam B. Pauli ad Philippenses commentarii... Anvers, 1637. 2 vol. - Ex. double. s.d. $/ 1745$

645 Le véritable esprit des nouveaux disciples de S. Augustin... Bruxelles, 1706. 3 vol.

(Donné pour rester dans les missions).

646 Verney, Joseph Guichard du, 1648-1730.

Traité de l'organe de l'ouie ... Leide, 1731.

1745

1745

Hôp. Gén.

647 Le véritable art du blason. 2 vol.

C

648 La véritable manière de prêcher.

$\mathrm{C}$

649 Vers solitaires et autres de diverses espèces ... Paris, 1718.

$1720 / 1745$

Hôp. Gén.

650 La vie de Jésus-Christ... Paris, 1678.

s.d./1745

Hôp. Gén.

651 La vie de la Mère Magdeleine de S. Joseph ... Paris, 1670.

$$
1745
$$

Hôp. Gén.

652 La vie de la Mère Térèse de Jésus fondatrice des religieuses et religieux Carmes deschaussés ... Paris, 1601.

1745

653 La vie de la vén. Mère Marie de l'Incarnation première supérieure des Ursulines de la Nouvelle-France. Paris, 1677 . s.d./1745

Hôp. Gén.

654 La vie de S. François de Borgia ... Paris, 1672.

$1720 / 1745$

Hôp. Gén.

655 Vie du Père Régis.

C 
656 Ville, Antoine de, 1596-1656.

Les fortifications ... Lyon, 1628.

657 Vimont, Barthelemy, s.j., 1594-1667. Relation... 1640-41.

658 Id.

Relation. 1642.

659 Id.

Relation. $1642 \& 1643$.

660 Virgile.

Oeuvres. Paris, 1716. 3 vol.

s.d./1745

661 Voragine, Giacomo de, 1230-1298.

Legenda sanctorum. Lyon, $1483 . \quad$ s.d./1745

662 Le vrai fidèle à Dieu et aux hommes. Paris, 1669 . 1720/1745

663 Wagret, J.-P.

Nouveau traité de la petite vérole. Paris, 1720.

1745

664 Wall, Jacques, s.j., 1599-1680.

Pomatum libri novem. Anvers, $1657 . \quad$ s.d./1745

665 Winslow, Jacques-Bénigne, 1669-1760.

Exposition anatomique de la structure du corps humain ... Paris, 1732. 3 vol.

1745

666 Zwelfer, Jean, 1618-1688.

Pharmacopeia augustana... Goudæ, 1653.

$1720 / 1745$ 


\section{INDEX DES MATIEERES}

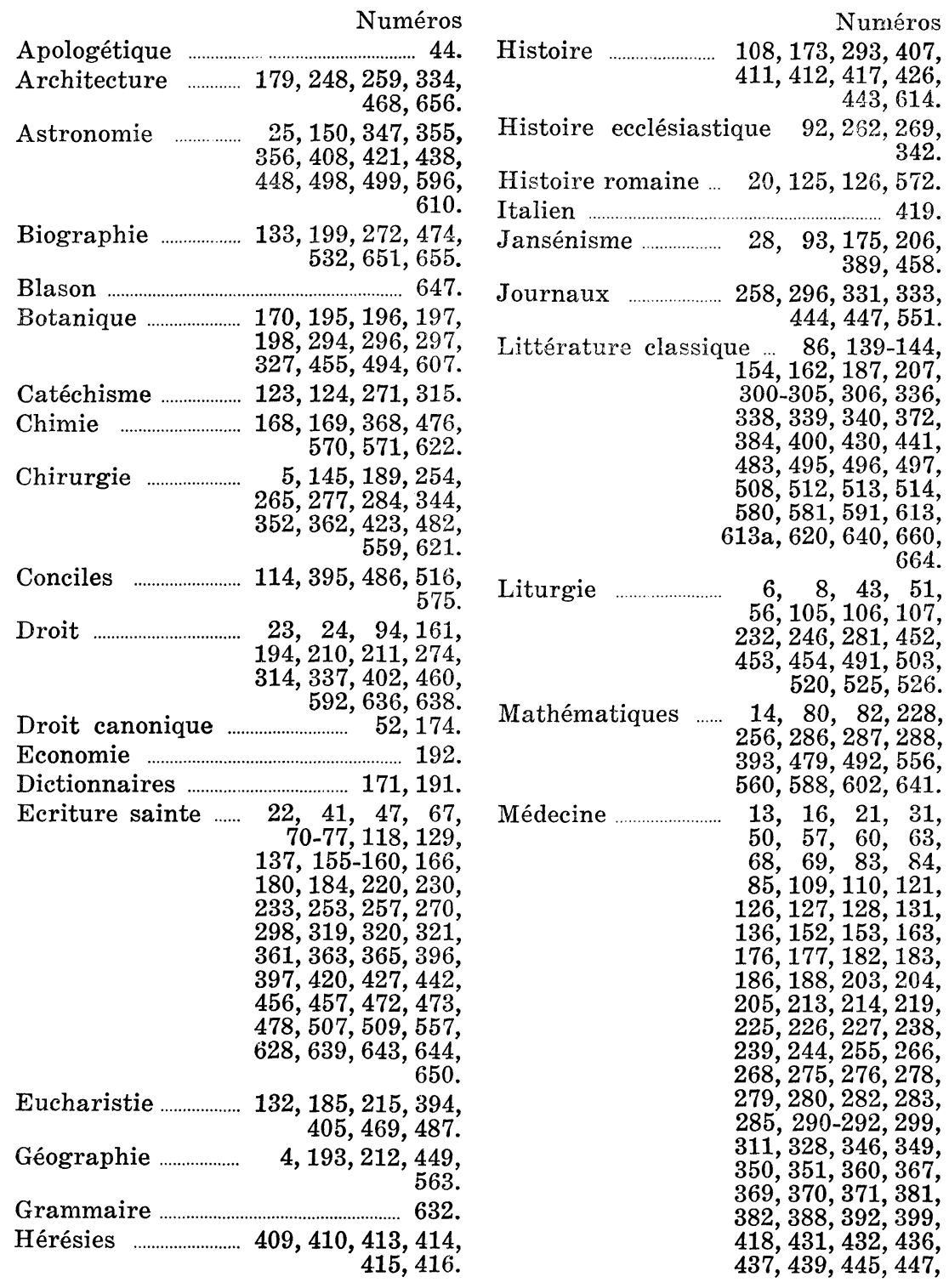




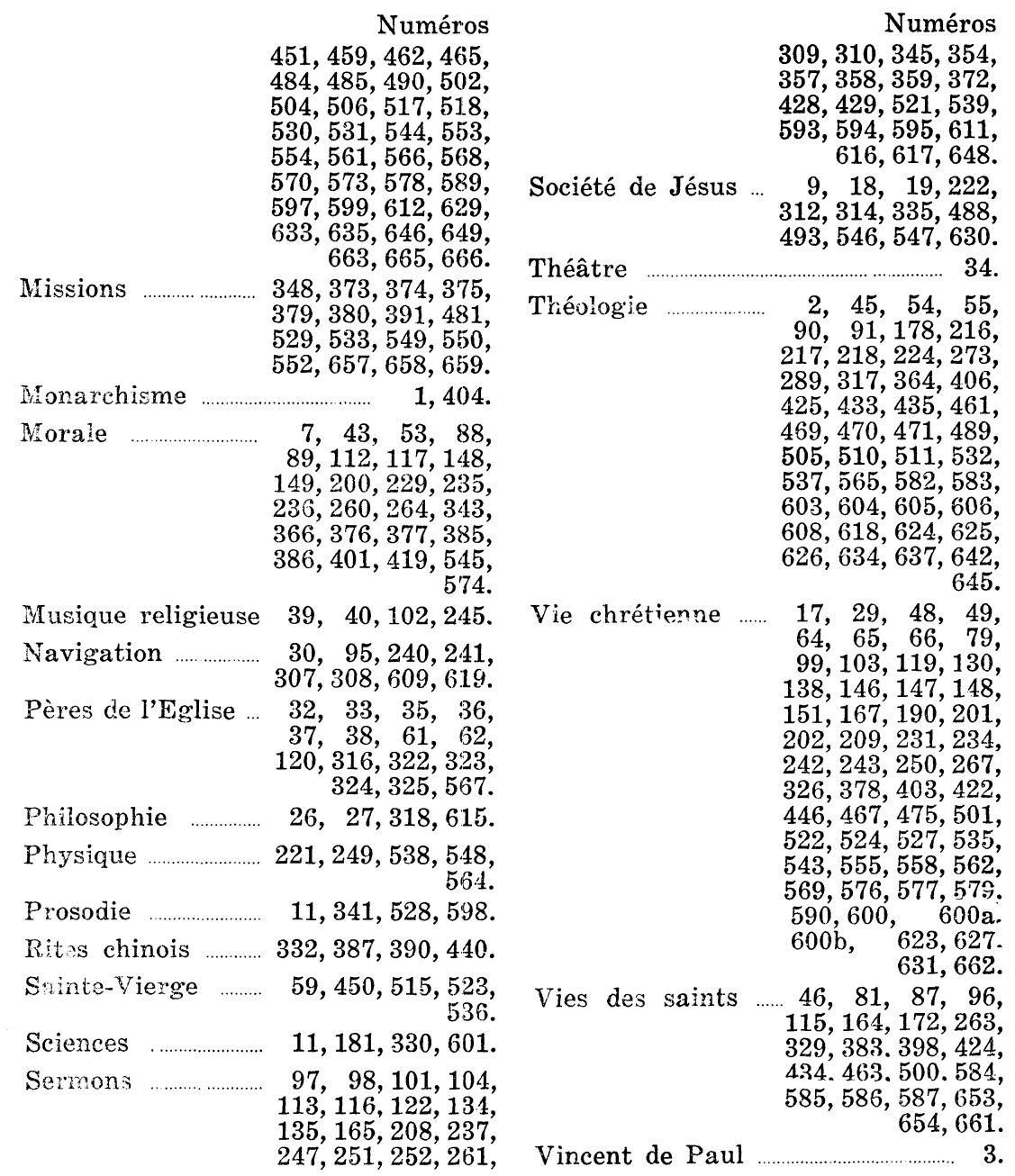

